\title{
Dynamic response of hybrid carbon fibre laminate beams under
}

\section{Abstract}

This novel hybrid fibre composites combining stiff composites with soft composites are developed to improve the ballistic impact resistance of composite beams while maintaining good quasi-static loading bearing capacity. The ballistic impact performance of the hybrid beams have been investigated experimentally at a projectile velocity range of $50 \mathrm{~ms}^{-1} \leq v_{0} \leq 300 \mathrm{~ms}^{-1}$, including ballistic limits, failure modes, energy absorption capacity and the interaction between stiff and soft composite parts. For each type of monolithic beams, i.e. stiff, soft and hybrid monolithic beams, three categories of failure modes have been identified: minor damage with rebound of projectile at the low impact velocities, fracture of beam at the medium impact velocities and perforation of beam at the high impact velocities. The critical velocity of hybrid monolithic beam was similar to that of the soft monolithic beam under the same failure mode, and higher than that of the stiff monolithic beam. For the sandwich beams with stiff, soft and hybrid face sheets, the failure modes were similar to those of the monolithic beams. Among the monolithic beams, the hybrid and soft monolithic beams exhibited better energy absorption capacity than the stiff monolithic beams. As for the sandwich beams, the hybrid-face sandwich beams absorbed more kinetic energy of projectile 
than the soft-face sandwich beams at higher projectile velocity. The advantages of the stiff/soft hybrid construction include: (i) at lower impact velocity, the soft composite part survived with

43 longitudinal wave speed $\sqrt{\frac{E_{f}}{\rho_{f}}}$ of fibres. This approach provides a guidance in development of negligible damage under impact; (ii) due to the buffer effect of the soft part at the front face, stress distribution within the stiff part of the hybrid monolithic beams is more uniform than that of the stiff monolithic beams.

Keywords: Fibre composites, hybrid beams, ballistic impact, failure modes, energy absorption capacity

\section{Introduction}

Fibre reinforced composites have been attractive in both military and civilian applications due to their outstanding mechanical properties [1]. It has been demonstrated that the lightweight structures made of fibre composites possess excellent performances to resist ballistic impact when the composites laminate is in $\left[0^{\circ} / 90^{\circ}\right]$ cross-ply lay-up [2, 3]. Cunniff [3] reported that the ballistic limit of fibre composites are proportionally increasing with the Cunniff velocity $c^{*}$ of the fibre filament and can be defined as follow

$$
c^{*}=\left(\frac{\sigma_{f} \varepsilon_{f}}{2 \rho_{f}} \sqrt{\frac{E_{f}}{\rho_{f}}}\right)^{1 / 3}
$$

where $\sigma_{f}$ and $\varepsilon_{f}$ are the tensile strength and failure strain of fibres, respectively, while $E_{f}$ and $\rho_{f}$ are the tensile Young's modulus and density of fibres, respectively. Thus, the Cunniff velocity $c^{*}$ is governed by two material properties, i.e. specific strain energy $\frac{\sigma_{f} \varepsilon_{f}}{2 \rho_{f}}$ and the fibre composites of high ballistic limit $[4,5]$. However, it does not give any insight into the effect of matrix on the ballistic impact response of fibre composites. Matrix has the functions of bonding fibre reinforcements together and transferring stress between them [6]. It can also 
protect fibres against abrasion as well as adverse environmental impacts. Though the matrix itself is unable to dissipate a large amount of energy, it has an indirect effect on the energy absorption of fibre composites via load transfer with the broken fibres. Lee et al. [7] argued that, compared with soft matrix, stiff matrix resulted in less deformation degree of fibre reinforcements and more significant stress concentration. In addition, the enhancement of fibre/matrix bonding strength reduces ballistic impact resistance of fibre composites. Ruijter et al. [8] analysed the effect of matrix stiffness, at the range of $10^{-4}$ to $4 \mathrm{GPa}$, on the ballistic impact protection of Twaron ${ }^{\circledR}$ fabric composites via a series of experimental measurements. They found that the ballistic limit of the composites strongly depended on the matrix stiffness, and the highest ballistic limit was achieved when the matrix stiffness was at the range of 0.01 to $1 \mathrm{GPa}$. Beyond the stiffness of $1 \mathrm{GPa}$, the matrix restricted the deformation of fibres, while the matrix was unable to provide enough adhesion to bond the fibres together if below the stiffness of 0.01 GPa. Karthikeyan et al. [9] investigated the effect of shear strength on the ballistic response of laminated composites, including cured and uncured carbon fibre composites, and polyethylene fibre composites $\left(\right.$ Dyneema $^{\circledR}$ ) with two different matrices. They reported that the Cunniff velocity failed to characterise the ballistic resistance of fibre composites, and the ballistic limit of the composites increased with decrease of shear strength of the matrix. The matrix with lower shear strength was able to relieve more stress gradient of cross-ply laminates through interlaminar shearing [10], thus a wider range of membrane stretching in each layer was achieved which ensured higher impact force resistance. It was reported that the soft matrix laminates failed progressively by tensile rupture of fibres under ballistic impact $[5,9]$.

Although the composites with soft matrix exhibit better ballistic performance than the ones with stiff matrix, it has limited ability to resist the out-of-plane bending force as well as inplane compression owing to microbuckling of fibres. Ashby and Brechet [11] proposed that 
the hybrid material, which was a combination of two or more materials, could superimpose the properties of each material and be multifunctional. It has been demonstrated that the hybrid composite laminates reinforced by two or more types of fibres can offer better ballistic performance than the laminates reinforced by only one type of fibre $[5,12-16]$. As reported by Pandya et al. [12], the ballistic limit was increased by adding E-galss fibre layers to carbon fibre composites compared to the pure carbon fibre composites with the same thickness. Bandaru et al. [13] investigated the different combinations of the fibre reinforced composites, namely, glass fibre, carbon fibre and Kevlar fibre composites. They found that the Kevlar composite laminate hybridized with carbon fibre layer possessed the best ballistic resistance, and the ballistic performance could be improved by increasing the toughness of composites. O'Masta et al. [5] investigated the penetration behaviour of the hybrid laminate combined two types of ultrahigh molecular weight polyethylene (UHMWPE) fibre composites both with soft matrix $\left(\right.$ Dyneema ${ }^{\circledR}$ ). They reported that the penetration resistance of the multi-layer laminates might benefit from the optimized stacking sequence of layers, i.e. the layer with higher compressive strength and lower impendence as front face, and the layer with higher tensile strength as back face. The existing research on ballistic impact of hybrid fibre composites mainly focuses on the effect of hybridization of different types of fibre reinforcements embedded in single type of matrix, i.e. either stiff or soft matrix. With regards to certain structures, the requirements for stiffness and ballistic resistance are equally important, e.g. the shell of an airplane nose subject to bird collision and the hood of an automobile subject to bullets as well as debris impact. Hence, it is imperative to develop hybrid composites which can not only guarantee structural stiffness but also resist high-velocity ballistic impact. However, limited ballistic impact tests have been reported on the fibre composites hybridizing stiff composite and soft composite, which may benefit from good ballistic resistance from the soft part and good quasi-static loading bearing capacity from the stiff part. As the failure modes 
of these two types of fibre composites are different, the mechanism of the interaction between these two composites has not been well established. Although Larsson et al. [15] gave an insight into the ballistic performance of the hybrid composites which combined stiff carbon fibre composites with soft polyethylene fibre composites, the failure modes as well as energy absorption capacity of the hybrid material containing soft and stiff composites are still unclear. Sandwich structures with fibre composite face sheets and honeycomb core are multi-functional lightweight structures owing to the good bending resistance and energy absorption capacity [17-19]. As the deformation are bending governed when they subject to soft impact, the fibre composite sandwich structures exhibit better soft impact resistance than the monolithic ones [20, 21]. However, Russell et al. [20] reported that the fibre composite beams failed catastrophically at a lower projectile impulse than the steel sandwich beams owing to the lower ductility of the fibre reinforced polymer composites. In addition, the ballistic impact resistance of sandwich structures improves negligibly compared to that of monolithic structures with the same areal mass. This is owing to the fact that the ballistic impact is a kind of localised impact that doesn't lead to significant bending of structure. It is inspired that replacing a part of stiff face sheets of sandwich structures with the soft composites may overcome these problems. The sandwich structures with stiff/soft hybrid face sheets are expected to not only prevent catastrophic failure of stiff face sheets under soft impact but also exhibit better resistance under ballistic impact. To date, the ballistic performance of this type of stiff/soft hybrid sandwich structure has not been investigated.

This paper experimentally investigates the ballistic impact response of a novel hybrid composite beam with stiff composites and soft composites, including the failure modes, energy absorption capacity, and the effect of the interaction between the stiff/soft composite parts on the deformation of hybrid beams. In the following sections of the paper, the experimental materials and manufacturing process are described in Section 2, and the mechanical properties 
of the constituent materials are presented in Section 3. In Section 4 and Section 5, the ballistic

123

124

125

126

127

128

129

130 impact methodology and experimental results are then discussed, respectively.

\section{Scope and novelty of this study}

The paper aims to investigate the ballistic impact performance of the novel stiff/soft hybrid fibre composite beams in comparison with those of stiff and soft monolithic beams. The novelty of this study includes

- The ballistic impact response of the hybrid fibre composite beams combining stiff composites and soft composites is experimentally measured at different impact velocities. The advantages of the hybrid beams are identified by comparing with the ballistic impact response of traditional monolithic beams from the aspects of ballistic limits, failure modes and energy absorption capacity.

- Both the ballistic resistance of novel stiff/soft hybrid monolithic beams and sandwich beams is investigated owing to the different bending stiffness and applications.

- The effect of time scales of wave propagation in stiff and soft composite parts on the failure modes and ballistic limits of beams is reported.

- The effect of the interaction between the stiff/soft composite parts on the deformation and failure mechanism of each part of hybrid beams is analysed.

\section{Materials and manufacturing}

\subsection{Materials}

The laminated composite sheets, used as the monolithic beams and face sheets of sandwich beams, were reinforced by Pyrofil TR50S 15K carbon fibres (diameter is $7 \mu \mathrm{m}$ ). The thickness of each unidirectional fibre layer was $0.1 \mathrm{~mm}$. The slow IN2 epoxy infusion resin and EF80 flexible epoxy resin, both supplied by Easy Composites Ltd, were used as the matrix materials for manufacturing different types of fibre composites. Both of them are two part (resin and hardener) epoxy resin system. The IN2 epoxy resin with low mixed viscosity (200-450 $\mathrm{mPa} \cdot \mathrm{s})$ 
147 is able to infuse through fibre reinforcements quickly, and becomes hard and brittle after full 148 cure. Hence, it is suitable for manufacturing resin infusion composites. As for the EF80 flexible 149 epoxy resin, it exhibits higher mixed viscosity (500-1200 $\mathrm{mPa} \cdot \mathrm{s})$ than the IN2 epoxy resin. In 150 addition, it has the capacity of maintaining flexibility after full cure, and is therefore suitable 151 for the applications where the flexibility of fibre reinforced composite parts are required. 152 Throughout the paper, the fibre composites with IN2 epoxy infusion resin are termed stiff 153 composites and the ones with EF80 flexible epoxy resin are termed soft composites.

Owing to the different bending stiffness and structural applications from those of monolithic composite beams, the sandwich beams were also investigated in this study. The phenolic resinimpregnated aramid paper honeycombs, commercially known as Nomex ${ }^{\circledR}$ honeycombs, were employed as the cores of the sandwich beams in this study owing to its high ratio of strength/stiffness to density [22-25]. The manufacturing process of the Nomex honeycombs is summarized as follow: the Nomex aramid paper layers made from random fibres are stacked on each other and adhered by the thermoset epoxy adhesive strips at intervals. The hexagonal unit cells were formed by expanding the paper layers along the stacking direction. Finally, the expanded geometry was impregnated into phenolic resin to be coated and obtain the specific density of the honeycombs. The density and out-of-plane thickness of the Nomex honeycomb core were $\rho_{h}=54 \mathrm{kgm}^{-3}$ and $H=10 \mathrm{~mm}$, respectively. Figure 1 (a) shows the in-plane structure of its hexagonal unit cell. The single-wall thickness of the unit cell geometry is $t_{h}=t_{f}+t_{r}$, where $t_{f}$ and $t_{r}$ are the thicknesses of the single aramid paper layer and phenolic resin layer, respectively. However, the wall thickness of the unit cell geometry along the stacking direction

168 is $2 t_{h}$ due to the expansion process. The characteristic cell size of the honeycombs is defined 169 as $L_{C}=\sqrt{3} L_{h}=4.8 \mathrm{~mm}$, with $L_{h}$ as the edge length of the hexagonal unit cell.

\subsection{Manufacturing}


In this study, both the stiff and soft fibre reinforced composite panels were manufactured using a vacuum assisted resin transfer moulding (VA-RTM) system. The unidirectional dry fibre layers were arranged in a $\left[0^{\circ} / 90^{\circ}\right]_{n}$ lay-up inside a mild steel mould, i.e. orthogonally stacking, as shown in Fig. 1 (b). The steel mould had one outlet port located at the centre and four inlet ports located at the four corners, both of diameter $2.5 \mathrm{~mm}$. Eight bolts at the edges of the mould were tightened to provide sufficient seal. Degassing of resin and gas tightness checking of VARTM system were conducted before resin injection. A vacuum pump connected with the outlet port created a vacuum environment in the mould to infuse the resin through the dry fibre layers. For soft matrix, the compressed air of pressure 8 bars within a catch-pot was imposed to facilitate the infusion of liquid resin. The ratios of resin to hardener by weight were $100: 30$ and $100: 145$ for manufacturing stiff composite panels and soft composite panels, respectively. The infused composite panels were then cured for $7 \mathrm{~h}$ at $65^{\circ} \mathrm{C}$. To reduce the flaws caused by cutting dry fibre layers, approximately $10 \mathrm{~mm}$ was removed from each edge of the panels after demoulding.

Figure 1 (c) shows the sketch of a Nomex honeycomb core sandwich beam specimen used for ballistic impact. Throughout this paper, the global coordinates are defined with the 3-axis aligned with the out-of-plane direction of beams, and with the 1-axis and 2-axis representing the in-plane directions of beams. Different types of face sheets used in sandwich beam specimens are listed in Fig. 1 (b). All the face sheets and monolithic beams of total length $L=240 \mathrm{~mm}$ and width $w=40 \mathrm{~mm}$ were cut from the cured laminated panels using a diamond saw, and the Nomex honeycomb core was cut by a sharp blade to be the same dimension as the laminated beams. The details of monolithic fibre composite beams (i.e. stiff monolithic beam, soft monolithic beam and hybrid monolithic beam) and sandwich beams (i.e. stiff-face sandwich beam, soft-face sandwich beam and hybrid-face sandwich beam) are summarized in Table 1. According to this Table, the stiff and soft composite beams of different 
thicknesses were used to assemble to form six types of beams with similar areal mass. $n$ in the

$197\left[0^{\circ} / 90^{\circ}\right]_{n}$ lay-up architecture is determined to be $10,5,4$ and 2 , respectively, corresponding to 198 the panel thickness of $t=3.9 \mathrm{~mm}, 1.9 \mathrm{~mm}, 1.6 \mathrm{~mm}$ and $0.8 \mathrm{~mm}$, respectively. The thicknesses 199 of the stiff and soft monolithic beams were both $t=3.9 \mathrm{~mm}$, and the thickness of each face 200 sheet of the stiff-face and soft-face sandwich beams were both $t=1.6 \mathrm{~mm}$. For the hybrid beams which comprised of stiff and soft composite parts with equal thickness, the thicknesses of each composite part of the hybrid monolithic beam and hybrid-face sandwich beam were $t=1.9 \mathrm{~mm}$ and $t=0.8 \mathrm{~mm}$, respectively. Hence, the number of fibre layer in sandwich beams was 4 less than that in monolithic beams. The fibre volume fractions and density of each laminated composite part were approximately $50 \%$ and $\rho=1380 \mathrm{kgm}^{-3}$, respectively.

Some additional steps were taken for assembling beams. The stiff and soft composite parts of 207 hybrid monolithic beams, and the face sheets of hybrid-face sandwich beams were glued together, respectively, using the Loctite EA $9461^{\circledR}$ epoxy adhesive. The face sheets and honeycomb cores of sandwich beams were glued together also using the Loctite EA $9461^{\circledR}$ epoxy adhesive. In hybrid-face sandwich beams, the part contacted with the each side of the Nomex honeycomb core was stiff part and soft part, respectively, as sketched in Table 1. In addition, to ensure the ends of the sandwich beams can be end-clamped sufficiently, the Nomex honeycomb core was filled with fast IN 2 epoxy resin, supplied by Easy Composites Ltd, over the clamped portion of each length $40 \mathrm{~mm}$. The assembled hybrid monolithic beams and sandwich beams were then cured in the oven for $5 \mathrm{~h}$ at $60{ }^{\circ} \mathrm{C}$ with $25 \mathrm{KN}$ transverse loading applied on the beams to achieve better bonding. The areal mass of the epoxy adhesive per layer

217 was measured to be $0.14 \mathrm{kgm}^{-2}$, and all the assembled composite beams had similar areal mass 218 in the range of $5.12-5.40 \mathrm{kgm}^{-2}$.

\section{Mechanical properties of the constituent materials}


221 The quasi-static uniaxial tensile and compressive responses of the fibre reinforced composites, and the quasi-static out-of-plane compressive response of Nomex honeycomb core were measured using an Instron screw-driven testing machine at an applied nominal strain rate $10^{-3}$

$224 \mathrm{~s}^{-1}$. There were five repeats for each type of test.

\subsection{Uniaxial tests on fibre reinforced composite sheet material}

The tension and compression tests on the stiff and soft fibre composite materials were conducted using the methods described by the EN ISO 527-4 and ASTM D3410/B, respectively. The aluminium tabs were adhered to the clamped ends of the rectangular specimens for friction gripping during test. The uniaxial forces of the specimens were determined by the load cell of the screw-driven testing machine, and the uniaxial strain of the specimens were measured by a single Stingray F-146B Firewire camera video gauge. In tension, the stiff and soft laminates both in $\left[0^{\circ} / 90^{\circ}\right]$ and $\pm 45^{\circ}$ orientations were tested. However, only the compressive response of the stiff laminate in $\left[0^{\circ} / 90^{\circ}\right]$ orientation was measured as the compressive response of the soft laminate in $\left[0^{\circ} / 90^{\circ}\right]$ orientations was too weak to be measured using the standard method. The specimens had a gauge length of $50 \mathrm{~mm}$ for tension test, whereas had a gauge length of $12 \mathrm{~mm}$ for compression test in order to prevent Euler buckling. Figure 2 (a) shows the measured nominal tensile and compressive stress versus strain relations of the composite laminates in $\left[0^{\circ} / 90^{\circ}\right]$ orientations. In the tension tests, the stiff and soft composite laminates displayed almost identical linear elastic responses, with the tensile strength of $535 \mathrm{MPa}$ and elastic modulus of $34 \mathrm{GPa}$. It was observed that the stiff and soft composite laminates had the same failure mechanism in tension, i.e. tensile fracture of fibre reinforcements. In the compressive tests, the stiff composites displayed elastic-brittle response, with the compressive strength was $221 \mathrm{MPa}$ at nominal strain of 0.011 .

244 In contrast, as the tensile response of the laminates in $\pm 45^{\circ}$ orientations was governed by the 245 shear of matrix, the laminates in this orientation were more ductile and had lower strengths 
than those in $\left[0^{\circ} / 90^{\circ}\right]$ orientations, see Fig. 2 (b). The stiff composites exhibit elastic-plastic

247 hardening response with the tensile strength of $187 \mathrm{MPa}$ and nominal failure strain of 0.225.

248 However, the soft composites have significantly lower tensile strength and higher nominal

249 failure strain, which are $36 \mathrm{MPa}$ and 0.36 , respectively in $\pm 45^{\circ}$ orientations. The slight

250 hardening response of the soft composites after initial yield is governed by the fibre rotation towards the tensile axis [9]. In addition, both the stiff and soft composites in $\pm 45^{\circ}$ orientations failed with matrix cracking without fibre rupture.

\subsection{Out-of-plane compression tests on Nomex honeycomb core}

The quasi-static out-of-plane compression tests on the Nomex honeycomb core were conducted using the same machine as that for testing the mechanical performance of fibre composite laminates. The tested honeycomb core specimen had an in-plane dimension of length $L_{L}=65$ mm and width $L_{W}=65 \mathrm{~mm}$, with 175 unit cells. The transverse load $F$ and deformation $\delta$ of honeycomb core were measured by the load cell and two symmetrically installed Linear Variable Differential Transformers (LVDT), respectively. The nominal compressive stress and strain of the specimen were taken as $\sigma=F / A$ and $\varepsilon=\delta / H$, respectively, with $A=65 \times 65 \mathrm{~mm}^{2}$ as the original cross-sectional area of the honeycomb core specimen. The measured nominal compressive stress versus strain curve of the honeycomb core is plotted in

Fig. 2 (c). It indicates that the specimen shows a linear elastic mechanical behaviour before achieving a peak compressive stress and has an abrupt softening after the peak stress, then displays hardening followed by densification at a nominal compressive strain of $\varepsilon=0.75$. The compressive strength of the Nomex honeycomb core was measured to be $\sigma_{S}=3.09 \mathrm{MPa}$.

\section{Ballistic impact test protocol}

Ballistic impact tests were conducted to investigate the failure modes and energy absorption capacity of the monolithic and sandwich composite beams, and find out the advantages of the hybrid beams. The sketch of the experimental setup developed by Turner et al. [26] is shown 
in Fig. 3. A steel fixture with four M6 bolts at each end was used for fully clamping the beams.

272 The fixture was fully fixed to minimize shock and guarantee negligible energy of projectile

273

274 transmitted to the fixture. Both the fixture and beams were put into a transparent polycarbonate cupboard to prevent projectile and debris of beams from flying out. The free span lengths of the beams were $170 \mathrm{~mm}$ and the front faces of beams were positioned $200 \mathrm{~mm}$ from the muzzle of the gas gun. The gas gun of barrel length $3.5 \mathrm{~m}$, outer diameter $16 \mathrm{~mm}$ and internal diameter $13 \mathrm{~mm}$ was employed for accelerating a non-deforming steel spherical projectile of diameter $d=12.7 \mathrm{~mm}$ and mass $M=8.3 \mathrm{~g}$. The calibration test before measurement was conducted to ensure the accelerated projectile impacted at the centre of the beams in all tests and no torsion occurred in the beams during impact. Either compressed air or pressurised liquid nitrogen was used to propel the projectile to various velocities in the range of $50 \mathrm{~ms}^{-1} \leq v_{0} \leq 300 \mathrm{~ms}^{-1}$, producing the initial kinetic energy of projectile in the range of $10.4 \mathrm{~J} \leq E_{\mathrm{k}_{-} 0} \leq 373.5 \mathrm{~J}$. The initial velocity of projectile was measured using two laser gates located at the open end of the gas gun barrel and confirmed with a Phantom Mercury HS v 12.1 high speed camera. The high speed camera was also used to capture the failure modes of beams and residual velocity of projectile during ballistic impact. Typically, the frame rate and exposure time were 38,000 fps and $10 \mu$ s, respectively, and the resolution was 320×344. The Dedolight Dedocool Standard 2light kit, which was able to concentrate an intense beam of light over a highly concentrated area, was set outside the polycarbonate cupboard to meet the requirements of high speed videography. In order to reflect more light into the high speed camera, a smooth aluminium panel was placed at the other side of beams, opposite to the camera. In addition, the cross sections of beams were painted to be white using marker pen for observing the deformation of beams more clearly. It should be noted that we suppose the soft composite parts, which are in hybrid monolithic and hybrid-face sandwich beams, act as a cushion that avoids the direct stiff 
contact between non-deforming projectile and stiff composite part. Based on this assumption, we set the projectile firstly impact the soft composites part of the hybrid beams.

297

298

\section{Results and discussion}

The experimental measurements for the six types of composite beams have been summarized in Table 2, including the initial projectile velocity, residual projectile velocity, kinetic energy of projectile transmitted to beams, and failure modes of beams.

\subsection{Impact responses of monolithic beams}

In this section, the responses of three types of monolithic composite beams under a series of ballistic impact tests were investigated, and the failure modes of these beams at various velocities are identified. The fracture mode discussed below is defined as the complete fracture of beams, and the perforation mode as the beams perforated without complete fracture. The critical velocity between two failure modes was calculated as the average value of the maximum velocity that triggered the low-velocity failure mode and the minimum velocity that triggered the high-velocity failure mode. Take the stiff monolithic beam for example, the measured maximum velocity for rebound mode was $56 \mathrm{~ms}^{-1}$, and the measured minimum velocity for fracture mode was $67 \mathrm{~ms}^{-1}$. Hence, the critical velocity between the rebound and fracture modes of the stiff monolithic beam was $61.5 \mathrm{~ms}^{-1}$.

\subsubsection{Stiff monolithic beam}

The back-face deflections of the stiff monolithic beams before failure as a function of time at selected impact velocities are plotted in Fig. 4. The back-face deflections of beams are measured through high speed photographs after ballistic impact. The montages of high-speed photographic images for three different failure modes are shown in Fig. 5 and discussed below Rebound $\left(v_{0} \leq 61.5 \mathrm{~ms}^{-1}\right)$ : The spherical projectile is rebounded by the deformed beam at impact velocity of $56 \mathrm{~ms}^{-1}$, as shown in Fig. 5 (a). 
Three-point fracture $\left(61.5 \mathrm{~ms}^{-1}<v_{0} \leq 116.5 \mathrm{~ms}^{-1}\right)$ : The beams fail with fibre fracture at three positions in this range of impact velocity. Figure 5 (b) shows that the fracture in the middle develops from the back face of the beam, thus the fracture mechanism is stretch governed. The fracture at the clamped ends is also stretch governed, as indicated in the photograph of Fig. 4. At impact velocity of $67 \mathrm{~ms}^{-1}$, the fracture mainly focuses on the middle of the beam while a part of fracture also occurs at the clamped ends (Fig. 4). At higher impact velocity of $100 \mathrm{~ms}^{-}$ ${ }^{1}$, the beam fully fractures at three points, i.e. middle and two clamped ends. The back-face deflection of the beam before fracture decreases with the increase of impact velocity. of projectile reaches to the perforation limit. As reported by Karthikeyan et al. [9], the projectile with high kinetic energy first comminutes the fibres at the impacted point, and then results in the local bending of back face. The significant bending of the back face leads to the tensile fracture of fibres (Fig. 5 (c)) and consequently the peroration of beam. The back face view of the perforated beam is diamond-shape damage at the impact point, as shown in Fig. 5 (c). The beams have been perforated before a large deflection achieves.

The failure modes and critical velocities of the stiff monolithic beams are similar to those of the three-dimensional woven carbon fibre resin composites [26].

\subsubsection{Soft monolithic beam}

337 The back-face deflections of soft monolithic beams before failure as a function of time history at selected impact velocities are plotted in Fig. 6, and the montages of high-speed photographic images for three different failure modes are shown in Fig. 7. As the beam has a long response history at low impact velocity of $72 \mathrm{~ms}^{-1}$, the response history at this velocity (Fig. 6 (a)) is

341 separated from others at higher velocities (Fig. 6 (b)) for clarity. The ballistic behaviour is described as follow 
343 Rebound $\left(v_{0} \leq 84 \mathrm{~ms}^{-1}\right)$ : At the velocity of $72 \mathrm{~ms}^{-1}$, the projectile is rebounded along with a 344 part of beam fracture in the width direction, as shown in Fig. 7 (a).

345 One-point fracture $\left(84 \mathrm{~ms}^{-1}<v_{0} \leq 232.5 \mathrm{~ms}^{-1}\right)$ : In this range of applied projectile velocity, the 346 soft monolithic beam only fractures in the middle. This is different from the three-point fracture 347 mode of the stiff monolithic beam. As shown in Figs. 7 (b) and (c), the beam is first partly 348 perforated by the projectile and then fully fractures in the middle. Fibre fracture along with 349 matrix cracking develops from the back face of the beam due to the significant bending at the 350 impact point.

351 Perforation $\left(v_{0}>232.5 \mathrm{~ms}^{-1}\right)$ : The beam is perforated without full fracture when the impact 352 velocity is high enough. The back-face deflection history of the beam for this failure mode is 353 not plotted in Fig. 6 as the deflection is negligible before perforation.

\subsubsection{Hybrid monolithic beam}

355 For the hybrid monolithic beam, the debonding occurs between the stiff and soft composite 356 parts during ballistic impact. The back-face deflections of both stiff part and soft part before 357 failure as a function of time are plotted in Fig. 8. As discussed in Section 4, the projectile 358 impacts the soft part firstly, then the stiff part in the back of the beam. The montages of highspeed photographic images for three different failure modes are shown in Fig. 9

360 Rebound $\left(v_{0} \leq 86 \mathrm{~ms}^{-1}\right)$ : The projectile was rebounded by the beam under low velocity impact, see Fig. 9 (a). Although the stiff composite part at the back face fractures, there is only slight cracking at the impacted surface of the soft composite part, as the micro photographs shown in

363 Fig. 9 (a). The hybrid monolithic beam can therefore still resist load after impact. Under the 364 same impact velocity, however, the stiff and soft monolithic beams are fractured fully and 365 partly, respectively, as discussed in Sections 5.1.1 and 5.1.2. 
One-point fracture $\left(86 \mathrm{~ms}^{-1}<v_{0} \leq 235 \mathrm{~ms}^{-1}\right)$ : Both the stiff and soft parts failed with beam

367 fracture in the middle, and the debonding developed from the impact point to the clamped ends.

368 During the ballistic impact, the fibre fracture is observed at the back face of the stiff part, see

369 Fig. 9 (b).

370 Perforation $\left(v_{0} \geq 235 \mathrm{~ms}^{-1}\right)$ : When the impact velocity is high enough, the projectile perforates

371 the beam with a negligible deflection. As shown in Fig. 9 (c), the debonding is not observed

372 before perforation, but develops after that. It is concluded that the debonding is due to the wave

373 propagation rather than the different stiffness of the stiff part and soft part. Unlike the stiff

374 monolithic beam in Fig. 5 (c), the back face view of the perforated beam at the impact point is

375 circle-shape damage. This is due to the transition effect of soft composites at the front face,

376 which results in more uniform stress distribution of the stiff composite sheet around the

377 projectile.

378 5.1.4. Discussion

379 Figure 10 shows a comparison of critical velocities with respect to the failure modes of stiff, soft and hybrid monolithic beams. The soft and hybrid monolithic beams have similar critical velocities regarding to the same failure mode, and both higher than the stiff monolithic beams, particularly for the failure mode of perforation.

383 The difference in critical velocities between the stiff and soft monolithic beams can be 384 explained as follow. The flexible and ductile EF80 epoxy matrix makes the soft monolithic beam more deformable and less brittle than the stiff composite beam, which contributes to longer interaction time between the projectile and composite beam. Hence, the plastic wave can keep propagating in the soft monolithic beam for the failure mode of fracture, even though the projectile has perforated the beam. With the increase of impact velocity, the interaction time and wave propagation time become shorter, and the beam finally fails with perforation when the perforation limit velocity reaches. Compared to the soft monolithic beam, the wave 
propagation time in stiff monolithic beam is shorter due to the stiffer interaction, and the projectile impacts on stiff monolithic beam is more likely to give rise to stress concentration.

393 Thus, the stiff monolithic beam can be perforated at lower impact velocity than the soft 394 monolithic beam. Throughout the impacts on stiff and soft monolithic beams, there is no 395 damage in terms of delamination observed in the plies.

396 Compared to the soft monolithic beam, the hybrid monolithic beam provides higher stiffness. The debonding between the stiff and soft parts of the hybrid monolithic beam can always be observed in the range of applied velocities, i.e. $72 \mathrm{~ms}^{-1}<v_{0} \leq 272 \mathrm{~ms}^{-1}$. Due to the high viscosity of the epoxy adhesive, the adhesive was unable to be degassed or vacuum infused, which resulted in more imperfections introduced in the adhesive. Hence, the debonding between the stiff and soft parts is easier to occur during impact. Based on the above analysis to the perforation mode of hybrid monolithic beams, the development of debonding is mainly governed by the wave propagation time in the beam, which is inversely scale with the initial velocity of projectile. Hence, at low and medium velocities, the long interaction time between the projectile and beam results in long wave propagation time and significant debonding (Fig. 9 (a) and (b)).

\subsection{Impact responses of sandwich beams}

The responses of sandwich beams with three types of face sheets, i.e. stiff face, soft face and hybrid face, respectively, under ballistic impact are investigated. The montages of high-speed photographic images at three impact velocity levels are shown in Fig. 11, Fig. 13 and Fig. 14.

411 At low impact velocity of approximately $73 \mathrm{~ms}^{-1}$, the projectiles are rebounded by the stiff412 face and soft-face sandwich beams, as shown in Figs. 11 (a) and (b). However, the projectile penetrates the front face sheet of the hybrid-face sandwich beam and reaches to the back face 414 sheet, leading to the debonding between back face sheet and honeycomb core, and finally trapped into the beam (Fig. 11 (c)). This may due to the fact that the initial kinetic energy of 
the projectile $\left(v_{0}=75 \mathrm{~ms}^{-1}\right)$ for hybrid-face sandwich beam is $7.8 \%$ higher than those of the

417 projectiles $\left(v_{0}=72 \mathrm{~ms}^{-1}\right)$ for stiff-face and soft-face sandwich beams. In addition, there is

418 debonding around the impact point occurred between front face sheet and honeycomb core of

419 the soft-face sandwich beam owing to the flexibility of soft composite sheet. As stated in

420 Section 2.2, though the number of fibre layer in sandwich beams is 4 less than that in monolithic

421 beams, all the sandwich beams are able to resist the projectiles and behave better than the stiff

422 and soft monolithic beams at this low velocity level. Figure 12 shows the back-face deflections

423 of monolithic and sandwich beams as a function of time at initial projectile velocity of approximately $73 \mathrm{~ms}^{-1}$. For clarity, only the deformation response of the soft composite part in hybrid monolithic beam before the fracture of stiff composite part is plotted. It indicates that the projectiles are rebounded by all the beams except for the stiff monolithic beam. The stiff monolithic beam fails with fully fracture, and the maximum deflection is $31 \mathrm{~mm}$ that is significantly higher than those (no more than $20 \mathrm{~mm}$ ) of other beams. The sandwich beams normally have smaller deflections than the monolithic beams due to the higher stiffness. At medium impact velocity of around $105 \mathrm{~ms}^{-1}$, the front face sheets of all sandwich beams are perforated and the back face sheets fully fracture during impact, as shown in Fig. 13. The debonding between back face sheet and honeycomb core is also observed in all sandwich beams. Similar to the hybrid monolithic beam, the sheet-sheet debonding occurs in the back face sheet of the hybrid-face sandwich beam, see Fig. 13 (c).

Figure 14 shows the montages of high-speed photographic images at higher impact velocity of around $144 \mathrm{~ms}^{-1}$. For the stiff-face sandwich beam, both the front face sheet and back face sheet are perforated without full fracture. The explanation to this is identical to that to the stiff monolithic beam, i.e. owing to the short interaction time between projectile and stiff composites. For the soft-face as well as hybrid-face sandwich beams, the failure modes are similar to those under the impact velocity of around $105 \mathrm{~ms}^{-1}$. 

projectile.

443 Figure 15 shows the initial projectile velocity $v_{0}$ as a function of residual projectile velocity $444 v_{r}$. Here, $v_{r}$ is assumed to be 0 when the projectile is trapped into the beam. The ballistic 445 impact resistance of the beams can be reflected by the slopes and intercepts of the fitting lines, i.e. higher slope and intercept correspond to better impact resistance of beams. This figure indicates that the lowest intercept and slope of fitting lines are from the stiff monolithic beam and stiff-face sandwich beam, respectively. In addition, the slopes of the stiff, soft and hybrid monolithic beams are higher than those of the corresponding stiff-face, soft-face and hybridface sandwich beams, respectively. This is because the number of fibre layer for monolithic beams is more than that for sandwich beams in order to achieve identical areal mass, and carbon fibre laminated composites play a far more significant role than the Nomex honeycomb core in resisting ballistic impact.

\subsection{Energy absorption capacity of beams}

The kinetic energy of the projectile transmitted to the beams can be calculated as follow

$$
\Delta E_{a b s}=E_{k_{-} 0}-E_{k_{-} r}=\frac{1}{2} M\left(v_{0}^{2}-v_{r}^{2}\right)
$$

where $E_{k_{-} 0}$ and $E_{k_{-} r}$ are the initial and residual kinetic energy of projectile, respectively. $\Delta E_{a b s}$ is the energy transmitted from the projectile to fibre composite beams. This transmitted energy converted to the kinetic energy of beams and energy absorbed by beams. Based on Fig. 15, the kinetic energy of projectile transmitted to beams as a function of initial kinetic energy of projectile is summarized in Fig. 16. The initial kinetic energy of projectile is in the range of $13 \mathrm{~J} \leq E_{k_{-} 0} \leq 307 \mathrm{~J}$. Due to the different architectures, the monolithic and sandwich beams may acquire different kinetic energy during the impact events. Assuming that the kinetic energy acquired is identical for the beams with the same architecture, i.e. monolithic or sandwich, 
during impact. As described in Section 4, the fixture for clamping beams was fully fixed, hence the energy of projectile absorbed by the fixture can be neglected. Hence, the energy absorbed by beams with the same architecture can be compared using the kinetic energy of projectile transmitted to beams.

469 For the monolithic beams, the soft and hybrid monolithic beams have the best energy absorption capacity, whereas the soft monolithic beam behaves better in energy absorption than the hybrid monolithic beam when the initial projectile velocity is higher than $160 \mathrm{~ms}^{-1}$, as highlighted in Fig. 16. The stiff monolithic beam behaves worst in energy absorption. For the sandwich beams, both the soft-face and hybrid-face sandwich beams exhibit better energy absorption capacity than stiff-face sandwich beams. As discussed in Section 5.1.3, the buffer of soft composite part resulted in more uniform and wider range of stress distribution in beams. Hence, more energy of projectile can be absorbed by the face sheets and honeycomb core of soft-face and hybrid-face sandwich beams than by those of stiff-face sandwich beams. As the failure mode of the soft-face sandwich beams is same in the impact velocity range of $107 \mathrm{~ms}^{-1}<v_{0} \leq 145 \mathrm{~ms}^{-1}$, the energy absorption capacity of these beams reaches a plateau. However, within this velocity range, the energy absorbed by hybrid-face sandwich beams still increasing. The hybrid-face sandwich beam has better energy absorption capacity than softface sandwich beam at impact velocity of $145 \mathrm{~ms}^{-1}$. This may due to the interaction between the soft and hard parts of hybrid face sheets. The other reason may be the debonding between back face sheet and honeycomb core, which absorbs a part of kinetic energy of projectile.

485 Except for the soft-face and hybrid-face sandwich beams, the measured maximum initial 486 kinetic energy of projectile regarding to the mode of fracture is marked in Fig. 16 using an 487 upward dash arrow. This kinetic energy can be regarded as the critical value that results in the transition of failure modes from fracture to perforation. It indicates that the energy absorption capacity of these beams normally decreases during the transition of these two failure modes. It 
can be explained as follow. Along the width direction of the beams, there are less fibres fracture

491 for the failure mode of perforation than those for the failure mode of fracture. As the energy

492

493

494

495

496

497

498

499

500

501

502

503

504

505

506

507

508

509

510

511

512

513

514 absorption capacity of composites is proportional to the failed fibres [7], the beams failed with perforation therefore absorb less kinetic energy of projectile than the beams failed with fracture. However, there is a slight increase for the energy absorbed by the stiff monolithic beam during the transition of failure modes. This is due to the fact that the stiff monolithic beam failed with perforation has wider range of fibre deformation and damage (e.g. fracture and comminution) than that failed with fracture. It can be demonstrated by comparing the high-speed photographic images in Figs. 6 (b) and (c), and also by Karthikeyan et al. [9]. This explanation is not suitable for the stiff-sheet sandwich beams as the beam failed with fracture of back face sheet also has significant fibre deformation and damage, as shown in Fig. 14 (a).

\subsection{The effect of epoxy adhesive}

Except for the failure of carbon fibre reinforcements, the epoxy adhesive also failed due to the debonding between stiff and soft composite parts as well as face sheet and honeycomb core. There are more debondings observed in hybrid monolithic and hybrid-face sandwich beams than the other types of beams. In the present study, the tensile strength of the adhesive is 30 MPa [27], much lower than that of the carbon fibre. Russell et al. [20] numerically demonstrated that no more than $5 \%$ of the initial kinetic energy of projectile is dissipated by the delamination of fibre layers in the soft impact events. Kirthikeyan and Russel [10] reported that the ballistic limit of the pre-delaminated fibre laminate was $10 \%$ higher than that of the laminate with same areal mass but without pre-delamination. This was due to the benefit of delamination that promoted an earlier transition from fibre fracture to stretching. The debonding, between the stiff and soft composite parts of hybrid beams, governed by the lowstrength adhesive can also be regarded as 'pre-delamination'. Hence, the weak adhesive interface may play an important role in indirectly dissipating impact energy of a projectile. 
516 The ballistic responses of six types of carbon fibre composite beams, i.e. three monolithic

517 beams and three sandwich beams, have been investigated to identify the advantages of hybrid

518 beams. For each type of monolithic beam, there were three distinct failure modes identified:

519 minor damage with projectile rebound, fracture and perforation. The failure modes of fracture

520 and perforation were mainly governed by the fracture of fibre reinforcements, and the

521 development of these two damage modes depended on the wave propagation time in beams.

522 The hybrid and soft monolithic beam had similar critical velocities for each failure mode, and

523 both higher than the stiff monolithic beam. In addition, the hybrid monolithic beam had benefits

524 under low velocity impact as the failure only occurred in the stiff composite part of beam and

525 the soft part could still resisting loading. The back face damage mode of the hybrid monolithic

526 beam that failed with perforation was different from that of stiff monolithic beam ascribed to

527 the buffer effect of the soft composite part at the front face. For the stiff-sheet, soft-sheet and

528 hybrid-sheet sandwich beams, the failure modes were similar to those of the corresponding

529 monolithic beams, i.e. the projectiles were rebounded by or trapped into sandwich beams at

530 low impact velocity, and the back face sheet fully fractured and were perforated at medium and

531 high impact velocities, respectively.

532 The energy absorption capacity of the monolithic and sandwich beams have also been studied.

533 For the monolithic beams, the energy absorption capacity of the hybrid and soft monolithic

534 beams were better than that of the stiff monolithic beams, whereas the stiff monolithic and

535 stiff-face sandwich beams behaved worst. In addition, as more fibre reinforcements fractured,

536 the beams failed with fracture had better energy absorption capacity than those failed with

537 perforation. The hybrid-face sandwich beams exhibited better energy absorption capacity than

538 the soft-face sandwich beams at high impact velocity. 
539 The weak adhesive interface between the stiff and soft composite parts in hybrid 540 monolithic/sandwich beams may have a positive effect on the energy absorption capacity of

541 beams. The strength and flexibility of adhesive may influence the development of debonding,

542 their effects on the ballistic impact resistance of hybrid laminated composites is a future topic.

\section{Acknowledgments}

544 The authors acknowledge the support from the Royal Society through Research Grant Scheme.

545 The first author is grateful for the financial support from the China Scholarship Council (CSC).

\section{Declaration of interest: none}

\section{References}

548 [1] Turner P, Liu T, Zeng X. Dynamic Response of Orthogonal Three-Dimensional Woven 549 Carbon Composite Beams Under Soft Impact. Journal of Applied Mechanics. 2015;82:121008. 550 [2] Cheeseman BA, Bogetti TA. Ballistic impact into fabric and compliant composite laminates. 551 Composite Structures. 2003;61:161-73.

552 [3] Cunniff PM. Dimensionless Parameters for Optimization of Textile-Based Body Armor 553 Systems. Proceedings of the 18th International Symposium on Ballistics. San Antonio1999.p. $554 \quad 1303-10$.

555 [4] O'Masta MR, Deshpande VS, Wadley HNG. Mechanisms of projectile penetration in 556 Dyneema ${ }^{\circledR}$ encapsulated aluminum structures. International Journal of Impact Engineering. $557 \quad 2014 ; 74: 16-35$.

558 [5] O’Masta MR, Crayton DH, Deshpande VS, Wadley HNG. Mechanisms of penetration in 559 polyethylene reinforced cross-ply laminates. International Journal of Impact Engineering. $560 \quad 2015 ; 86: 249-64$.

561 [6] Congress US. Advanced materials by design. Washington, DC1988.

562 [7] B.L. Lee, Walsh TF, Won ST, Patts HM. Penetration failure mechanisms of armor-grade 563 fiber composites under impact. Journal of Composite Materials. 2001;35:1605-33. 
564 [8] de Ruijter C, van der Zwaag S, Stolze R, Dingemans TJ. Liquid crystalline matrix polymers 565 for aramid ballistic composites. Polymer Composites. 2010;31:612-9.

566 [9] Karthikeyan K, Russell BP, Fleck NA, Wadley HNG, Deshpande VS. The effect of shear 567 strength on the ballistic response of laminated composite plates. European Journal of 568 Mechanics - A/Solids. 2013;42:35-53.

569 [10] Karthikeyan K, Russell BP. Polyethylene ballistic laminates: Failure mechanics and 570 interface effect. Materials \& Design. 2014;63:115-25.

571 [11] Ashby MF, Bréchet YJM. Designing hybrid materials. Acta Materialia. 2003;51:5801-21.

572 [12] Pandya KS, Pothnis JR, Ravikumar G, Naik NK. Ballistic impact behavior of hybrid 573 composites. Materials \& Design. 2013;44:128-35.

574 [13] Bandaru AK, Vetiyatil L, Ahmad S. The effect of hybridization on the ballistic impact 575 behavior of hybrid composite armors. Composites Part B: Engineering. 2015;76:300-19.

576 [14] Bandaru AK, Ahmad S, Bhatnagar N. Ballistic performance of hybrid thermoplastic 577 composite armors reinforced with Kevlar and basalt fabrics. Composites Part A: Applied 578 Science and Manufacturing. 2017;97:151-65.

579 [15] Larsson F, Svensson L. Carbon, polyethylene and PBO hybrid fibre composites for 580 structural lightweight armour. Composites Part B: Engineering. 2002;33:221-31.

581 [16] Dorey G, Sidey GR, Hutchings J. Impact properties of carbon fibre/Kevlar 49 fibre hybrid 582 composites. Composites. 1978:25-32.

583 [17] Reyes Villanueva G, Cantwell WJ. The high velocity impact response of composite and 584 FML-reinforced sandwich structures. Composites Science and Technology. 2004;64:35-54.

585 [18] Wadley H. Fabrication and structural performance of periodic cellular metal sandwich 586 structures. Composites Science and Technology. 2003;63:2331-43.

587 [19] Gibson LJ, Ashby MF. Cellular solids: structure and properties. Cambridge, UK: 588 Cambridge University Press, 1997. 
589 [20] Russell BP, Liu T, Fleck NA, Deshpande VS. The soft impact of composite sandwich 590 beams with a square-honeycomb core. International Journal of Impact Engineering. $591 \quad 2012 ; 48: 65-81$.

592 [21] Tagarielli VL, Deshpande VS, Fleck NA. The dynamic response of composite sandwich 593 beams to transverse impact. International Journal of Solids and Structures. 2007;44:2442-57.

594 [22] Bourbigot S, Flambard X. Heat resistance and flammability of high performance fibres: 595 A review. Fire and Materials. 2002;26:155-68.

596 [23] Kandola BK, Krishnan L, Deli D, Ebdon JR. Blends of unsaturated polyester and phenolic 597 resins for application as fire-resistant matrices in fibre-reinforced composites. Part 2: Effects 598 of resin structure, compatibility and composition on fire performance. Polymer Degradation 599 and Stability. 2015;113:154-67.

600 [24] Zhou J, Yao Z, Chen Y, Wei D, Wu Y, Xu T. Mechanical and thermal properties of 601 graphene oxide/phenolic resin composite. Polymer Composites. 2013;34:1245-9.

602 [25] Zhang Y, Liu T, Tizani W. Experimental and numerical analysis of dynamic compressive 603 response of Nomex honeycombs. Composites Part B: Engineering. 2018;148:27-39.

604 [26] Turner P, Liu T, Zeng X, Brown K. Three-dimensional woven carbon fibre polymer 605 composite beams and plates under ballistic impact. Composite Structures. 2018;185:483-95.

606 [27] Loctite. http://www.loctite.co.uk/loctite-4087.htm?nodeid=8802629156865 2018.

607

608

609

610

611

612 
615 Figure 1. (a) The in-plane sketch of the Nomex honeycomb core unit cell in sandwich beam,

616 (b) the layer-up orientation of the fibre composite laminate and the types of face sheets. The

617 sketch of the assembled sandwich beam is shown in (c). The co-ordinate systems associated

618 with the beam and core are included in this figure. All dimensions are in $\mathrm{mm}$.

619 Figure 2. Quasi-static stress-strain relationships of the stiff and soft fibre composites under 620 uniaxial compression and tension tests for (a) $0^{\circ} / 90^{\circ}$ and (b) $\pm 45^{\circ}$ lay-up architecture. The measured quasi-static out-of-plane compressive response of the Nomex honeycomb core of 622 density $\rho=54 \mathrm{~kg} \cdot \mathrm{m}^{-3}$ is shown in (c).

623 Figure 3. Sketch of the experimental setup for ballistic impact on monolithic and sandwich 624 beams. All dimensions are in $\mathrm{mm}$.

625 Figure 4. The time history of back face deflection of the stiff monolithic beams at selected 626 impact velocities. Time $t=0$ corresponds to the time instant when the projectile impacted on the beams. The photographic image shows the part fracture of clamped end when the impact velocity was $67 \mathrm{~ms}^{-1}$.

629 Figure 5. Montage of the high speed photographs of the stiff monolithic beams under ballistic 630 impact. Three different failure modes of the beams are shown in this figure. The back face view of the beam failed with perforation is also shown in (c).

632 Figure 6. The time history of back face deflection of the soft monolithic beams at (a) impact 633 velocities of $72 \mathrm{~ms}^{-1}$ and (b) higher impact velocities. Time $\mathrm{t}=0$ corresponds to the time instant 634 when the projectile impacted on the beams.

635 Figure 7. Montage of the high speed photographs of the soft monolithic beams under ballistic 636 impact. Three different failure modes of the beams are shown in this figure. 
637 Figure 8 . The time history of back face deflections for stiff and soft parts in hybrid monolithic

638

639

640

641

642

643

644

645

646

647

648

649

650

651

652

653

654

655

656

657

658

659

660 beams at selected impact velocities. Time $\mathrm{t}=0$ corresponds to the time instant when the projectile impacted on the beams.

Figure 9. Montage of the high speed photographs of the hybrid monolithic beams under ballistic impact. Three different damage modes of the beams are shown in this figure. (a) also shows the micro damage of the stiff composites and soft composites after impact, and (c) also shows the back face view of the beam failed with perforation at the impact point.

Figure 10. The ranges of impact velocity regarding to the different damage modes of the stiff, soft and hybrid monolithic beams.

Figure 11. Montage of the high speed photographs of the (a) stiff-face, (b) soft-face and (c) hybrid-face sandwich beams impacted by the spherical projectile at velocity around $73 \mathrm{~ms}^{-1}$. The two red curves in (c) represent the edges of back face sheet and honeycomb core, and the front face view of hybrid-face sandwich beam at impact point are also shown in (c).

Figure 12. The time history of back face deflection for monolithic and sandwich beams at impact velocity of around $73 \mathrm{~ms}^{-1}$. It should be noted that the stiff monolithic beam and hybridface sandwich beam are impacted at the velocity of $67 \mathrm{~ms}^{-1}$ and $75 \mathrm{~m} \mathrm{~ms}^{-1}$, respectively.

Figure 13. Montage of the high speed photographs of the (a) stiff-face, (b) soft-face and (c) hybrid-face sandwich beams impacted by the projectile at velocity around $105 \mathrm{~ms}^{-1}$.

Figure 14. Montage of the high speed photographs of the (a) stiff-face, (b) soft-face and (c) hybrid-face sandwich beams impacted by the projectile at velocity around $144 \mathrm{~ms}^{-1}$.

Figure 15. Initial projectile velocity $v_{0}$ as a function of residual projectile velocity $v_{r}$. The projectile trapped in the hybrid-face sandwich beam has been highlighted in Fig. 11 (c). The straight dash lines are reference lines. The impact direction of projectile is along 3-axis of the coordinate system. 
661

662 energy of projectile.

663

664

665

666

Table 1. Details of the monolithic and sandwich beams.

\begin{tabular}{|c|c|c|c|c|c|c|}
\hline \multirow[b]{2}{*}{$\begin{array}{l}\text { Composite } \\
\text { sheets }\end{array}$} & \multicolumn{3}{|c|}{ Monolithic beams } & \multicolumn{3}{|c|}{ Sandwich beams } \\
\hline & Stiff & Soft & Hybrid & $\begin{array}{l}\text { Stiff face } \\
\text { sheet }\end{array}$ & $\begin{array}{c}\text { Soft face } \\
\text { sheet }\end{array}$ & $\begin{array}{c}\text { Hybrid } \\
\text { face } \\
\text { sheet }\end{array}$ \\
\hline Sketch of beams & & $\square$ & $\rightleftarrows$ & एणाए & एणाण & एणा \\
\hline $\begin{array}{l}\text { Number of } \\
\text { sheet layers }\end{array}$ & 20 & 20 & $10 * 2$ & $8 * 2$ & $8 * 2$ & $4 * 4$ \\
\hline $\begin{array}{c}\text { Areal mass of } \\
\text { laminates } \\
\left(\mathrm{kg} / \mathrm{m}^{2}\right)\end{array}$ & 5.38 & 5.38 & 5.30 & 4.30 & 4.30 & 4.30 \\
\hline $\begin{array}{c}\text { Areal mass of } \\
\text { honeycomb core } \\
\left(\mathrm{kg} / \mathrm{m}^{2}\right)\end{array}$ & 0 & 0 & 0 & 0.54 & 0.54 & 0.54 \\
\hline $\begin{array}{c}\text { Areal mass of } \\
\text { adhesive } \\
\left(\mathrm{kg} / \mathrm{m}^{2}\right)\end{array}$ & 0 & 0 & 0.14 & 0.28 & 0.28 & 0.56 \\
\hline $\begin{array}{c}\text { Total areal mass } \\
\text { of beams } \\
\left(\mathrm{kg} / \mathrm{m}^{2}\right)\end{array}$ & 5.38 & 5.38 & 5.44 & 5.12 & 5.12 & 5.40 \\
\hline
\end{tabular}

667

668

669

670

671

672

673

674

675 
Table 2 A summary of the experimental measurements for six types of composite beams.

\begin{tabular}{|c|c|c|c|c|}
\hline Beams & $\begin{array}{c}\text { Initial } \\
\text { velocity, } \\
v_{0}(\mathrm{~m} / \mathrm{s})\end{array}$ & $\begin{array}{l}\text { Residual } \\
\text { velocity, } \\
v_{r}(\mathrm{~m} / \mathrm{s})\end{array}$ & $\begin{array}{c}\text { Kinetic energy } \\
\text { transmitted to } \\
\text { beams, } \\
\Delta E_{a b s}(\mathrm{~J})\end{array}$ & Failure modes \\
\hline \multirow{5}{*}{$\begin{array}{l}\text { Stiff monolithic } \\
\text { beam }\end{array}$} & 56 & -16 & 11.95 & Rebound \\
\hline & 67 & -18 & 17.28 & Three-point fracture \\
\hline & 100 & 69 & 21.74 & Three-point fracture \\
\hline & 133 & 111 & 22.28 & Perforation \\
\hline & 160 & 136 & 26.75 & Perforation \\
\hline \multirow{5}{*}{$\begin{array}{l}\text { Soft monolithic } \\
\text { beam }\end{array}$} & 72 & -6 & 20.19 & Rebound \\
\hline & 96 & 26 & 35.44 & One-point fracture \\
\hline & 140 & 85 & 51.36 & One-point fracture \\
\hline & 207 & 153 & 80.67 & Three-point fracture \\
\hline & 258 & 220 & 75.37 & Perforation \\
\hline \multirow{5}{*}{$\begin{array}{c}\text { Hybrid } \\
\text { monolithic } \\
\text { beam }\end{array}$} & 72 & -8 & 21.25 & Rebound \\
\hline & 100 & 40 & 34.86 & One-point fracture \\
\hline & 145 & 86 & 56.56 & One-point fracture \\
\hline & 198 & 153 & 65.55 & One-point fracture \\
\hline & 272 & 246 & 55.90 & Perforation \\
\hline \multirow{3}{*}{$\begin{array}{c}\text { Stiff-face } \\
\text { sandwich beam }\end{array}$} & 72 & -14 & 21.70 & Rebound \\
\hline & 107 & 61 & 32.07 & Back face fracture \\
\hline & 145 & 124 & 23.44 & Back face perforation \\
\hline \multirow{3}{*}{$\begin{array}{c}\text { Soft-face } \\
\text { sandwich beam }\end{array}$} & 72 & -8 & 21.25 & Rebound \\
\hline & 107 & 43 & 39.84 & Back face fracture \\
\hline & 145 & 148 & 38.85 & Back face fracture \\
\hline \multirow{3}{*}{$\begin{array}{c}\text { Hybrid-face } \\
\text { sandwich beam }\end{array}$} & 75 & 0 & 23.34 & Projectile trapped \\
\hline & 100 & 42 & 34.18 & Back face fracture \\
\hline & 143 & 98 & 45.01 & Back face fracture \\
\hline
\end{tabular}




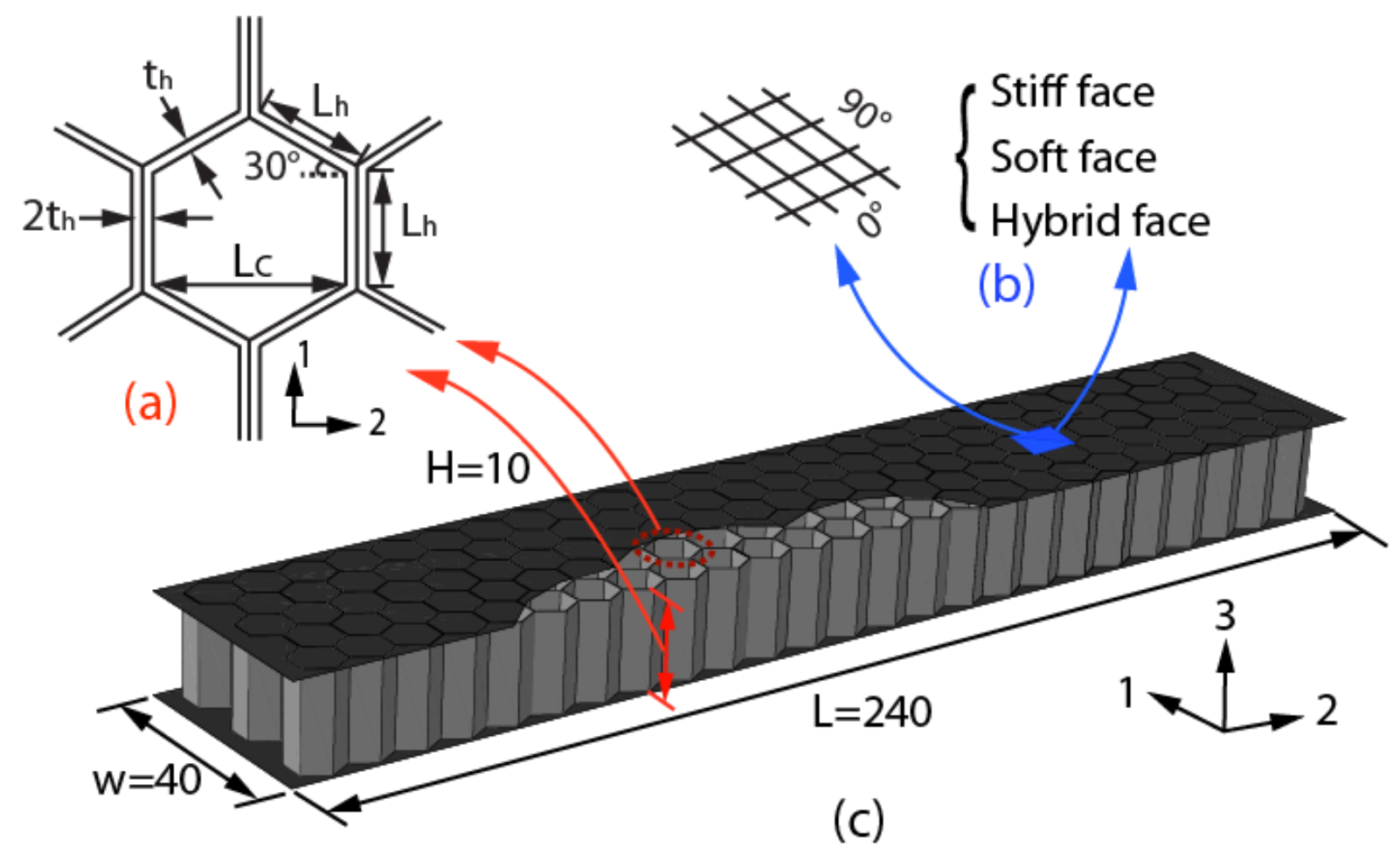

681

682

Figure 1

683

684

685

686

687

688

689

690

691

692

693

694

695 

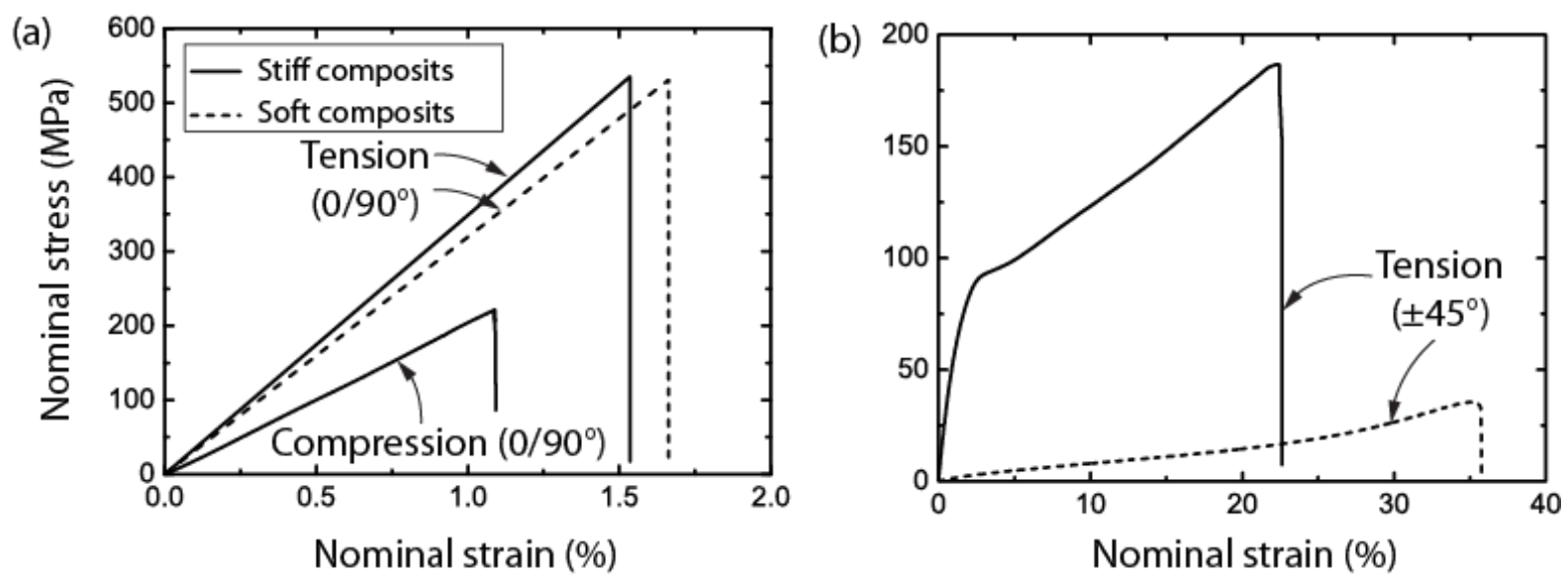

696

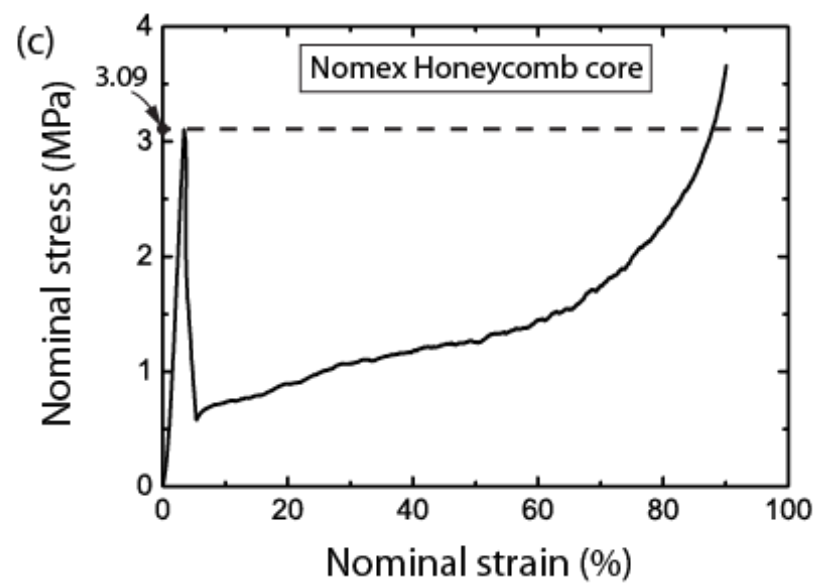

697

Figure 2

698 


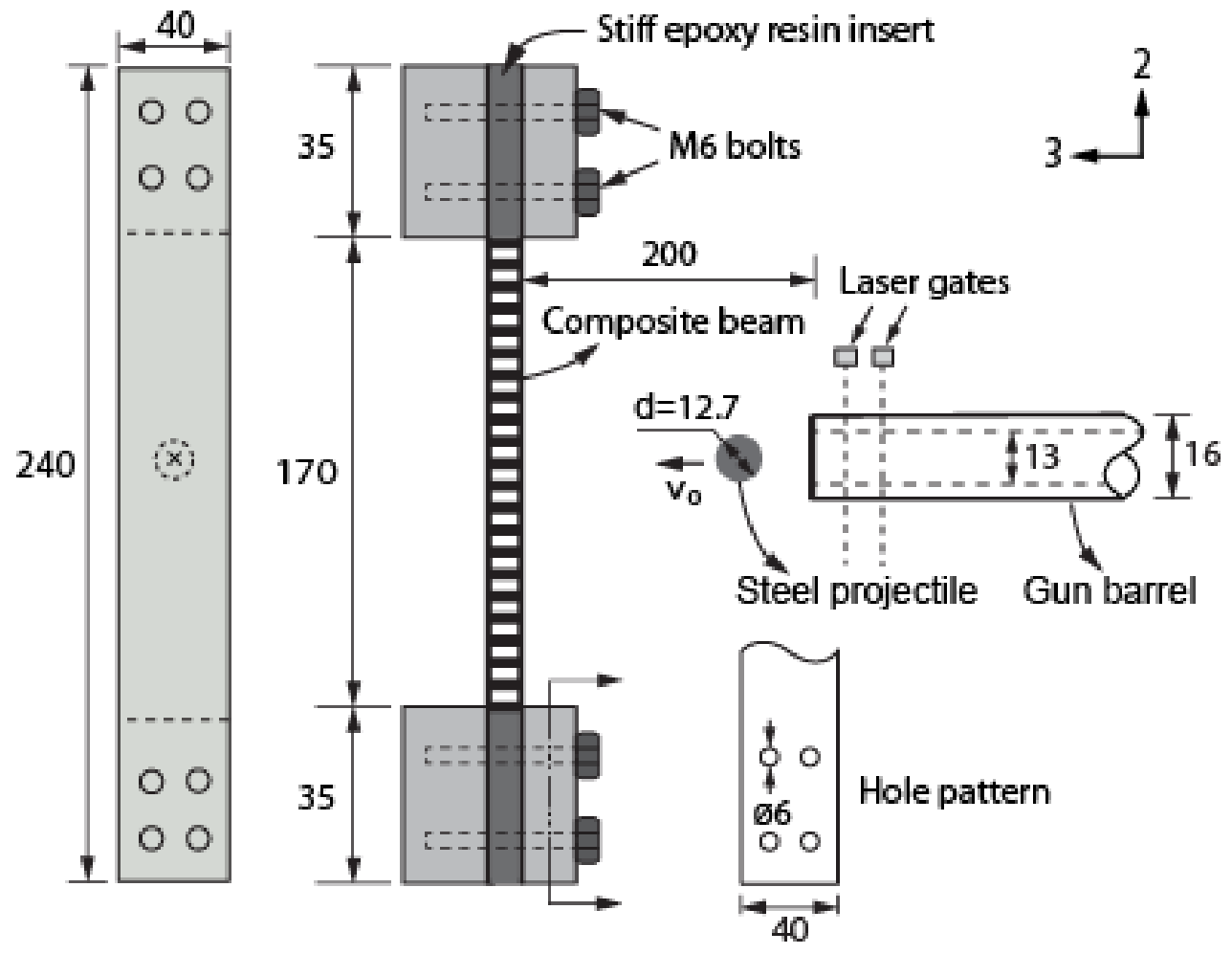

Figure 3

701

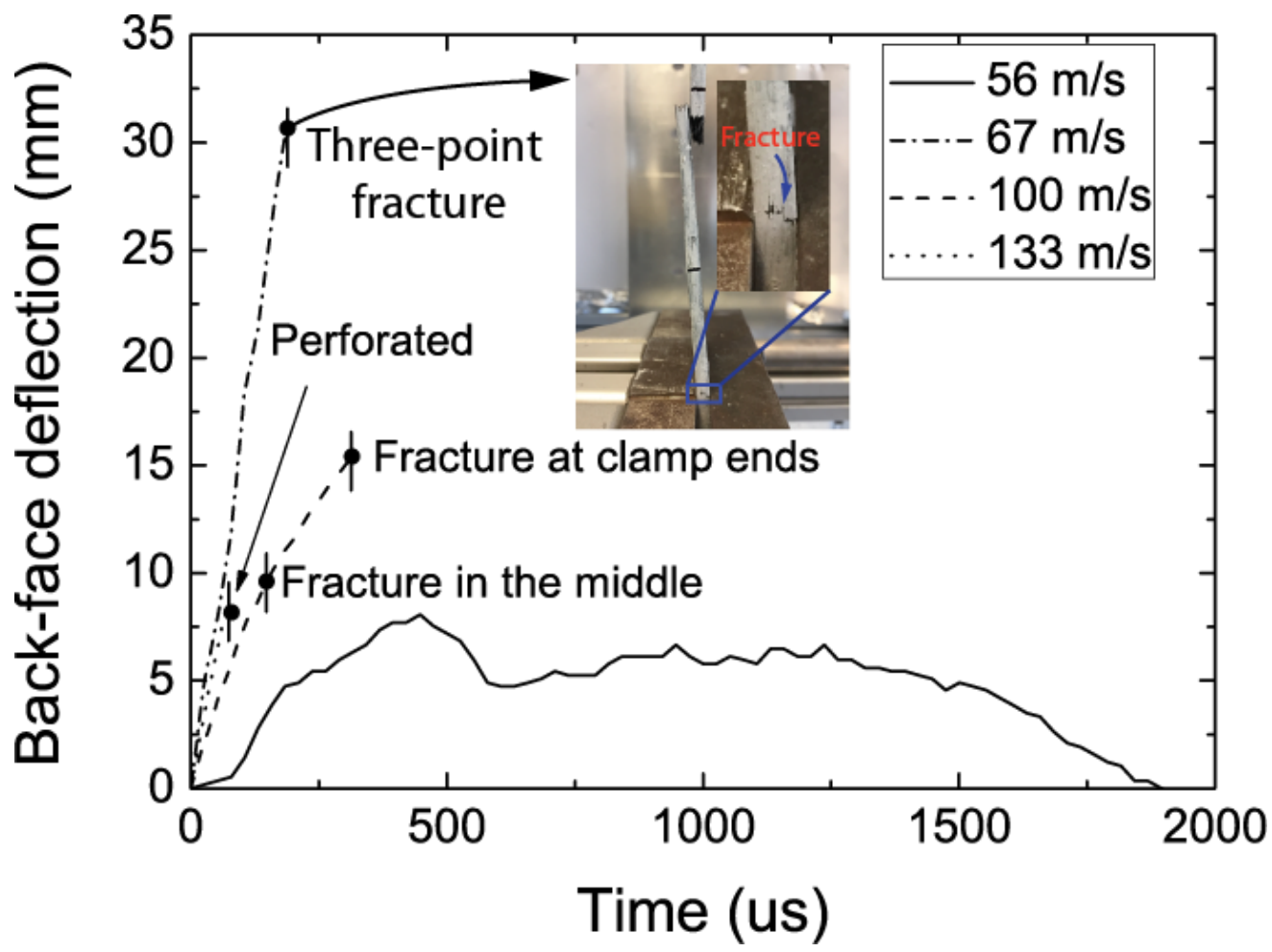

Figure 4 


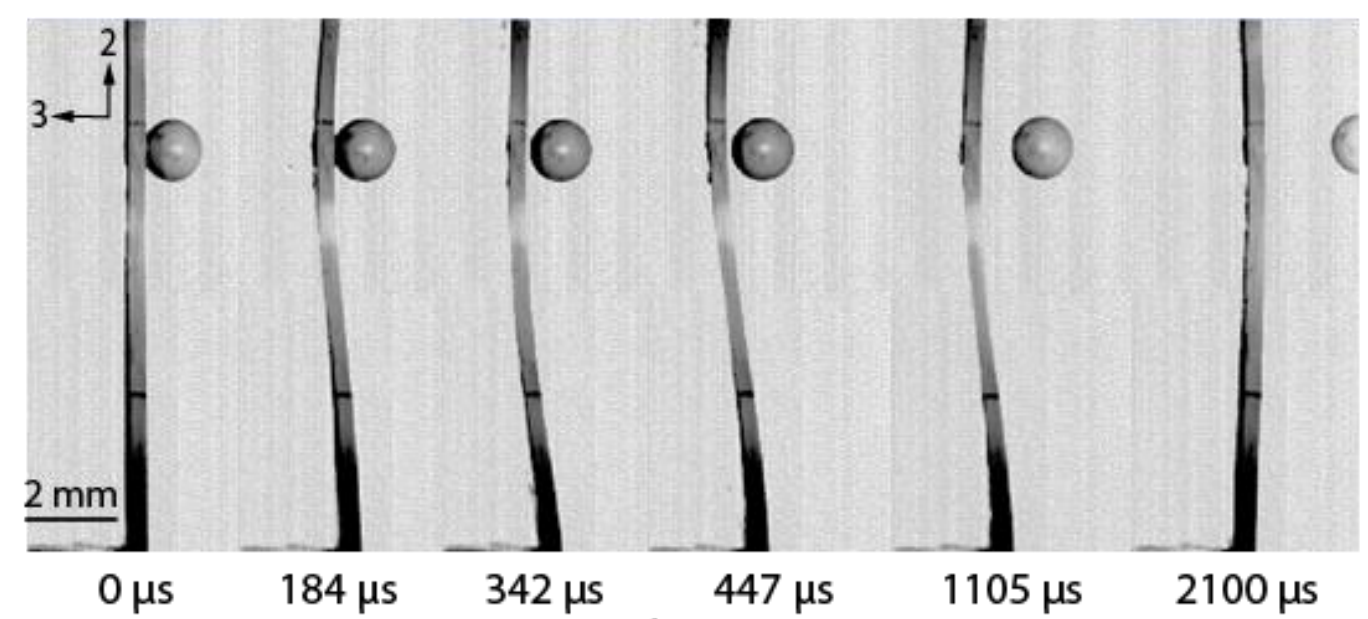

(a) $56 \mathrm{~ms}^{-1}{ }_{\text {Rebound }}$

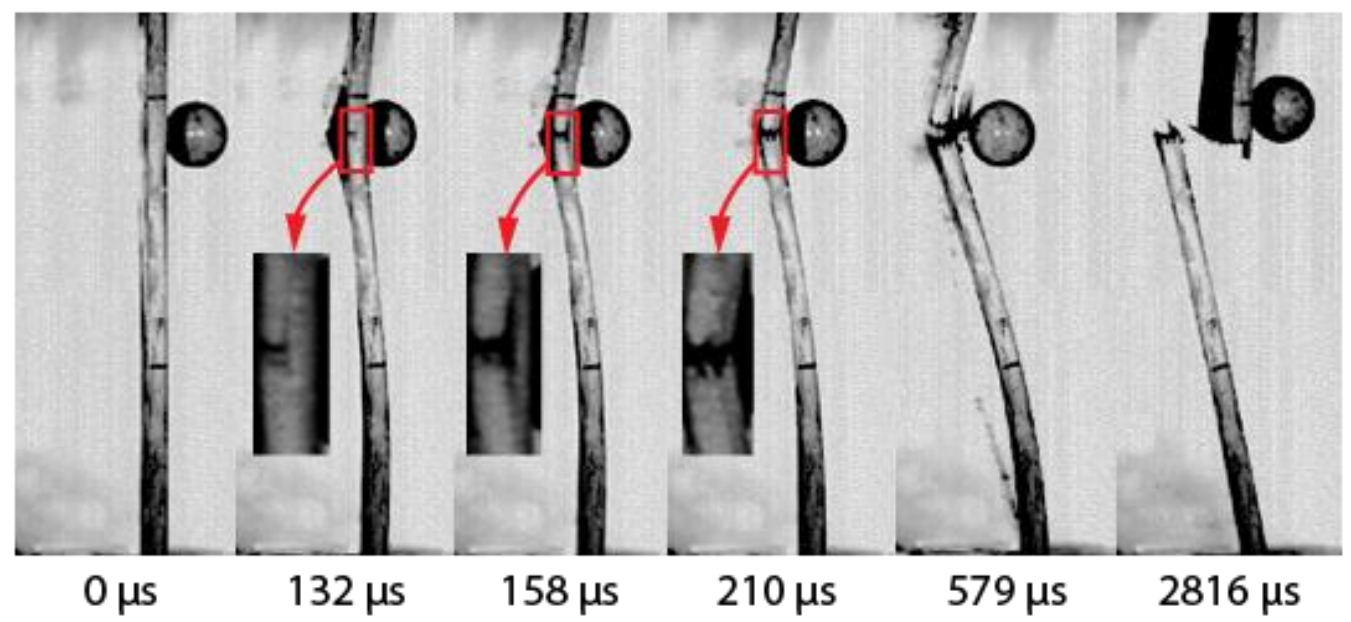

(b) $67 \mathrm{~ms}^{-1}{ }_{-}$Three-point fracture

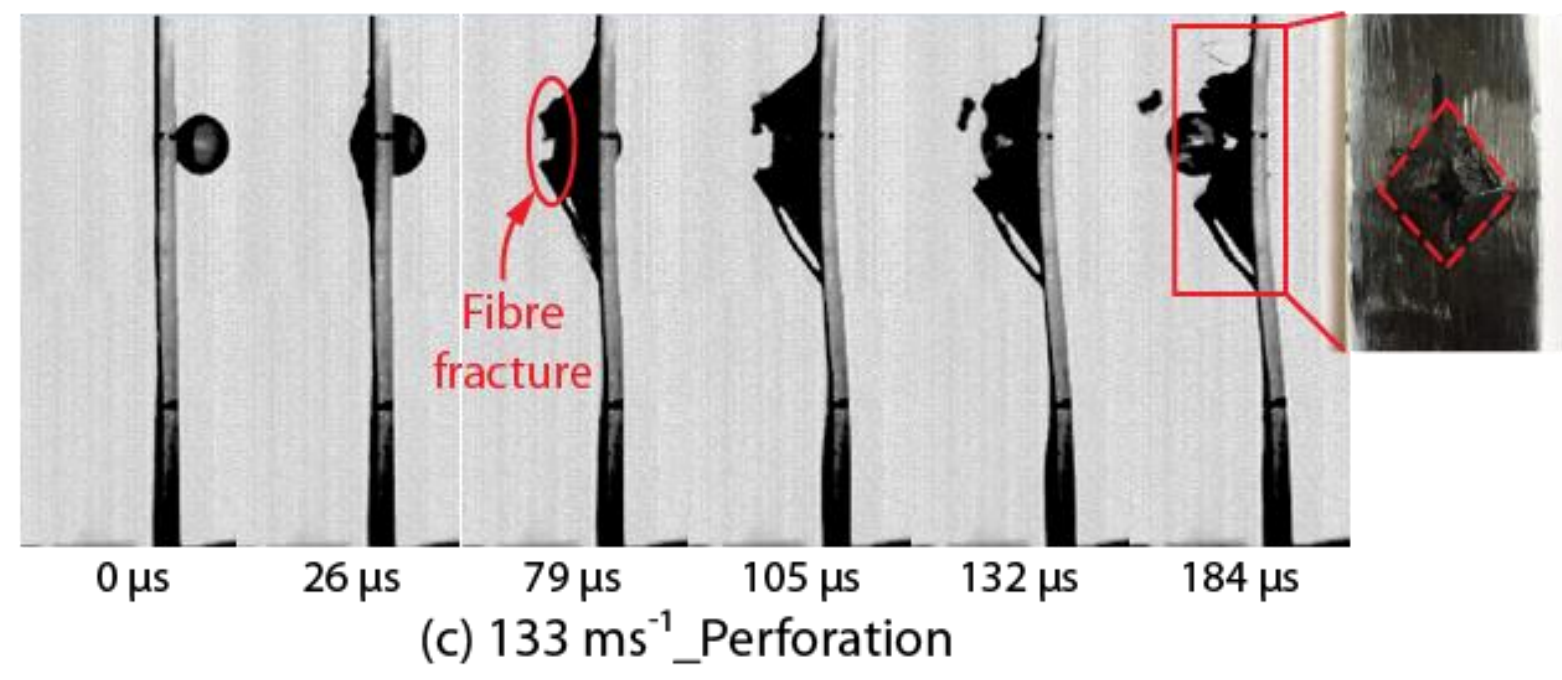

706

Figure 5

707

708 


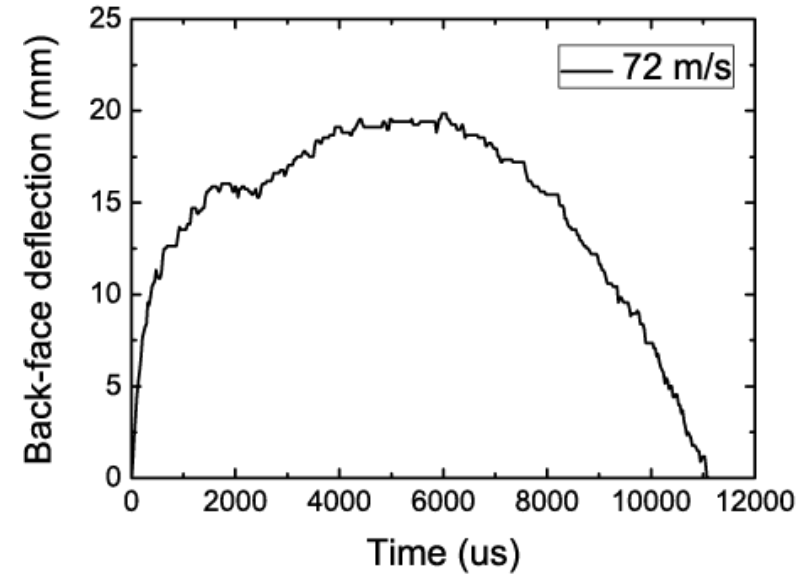

(a)

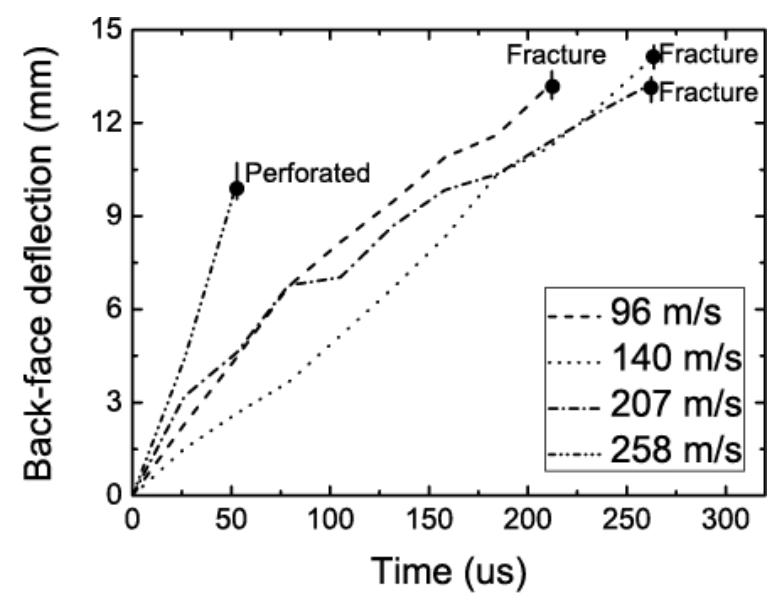

(b)

710

711

Figure 6

712

713 


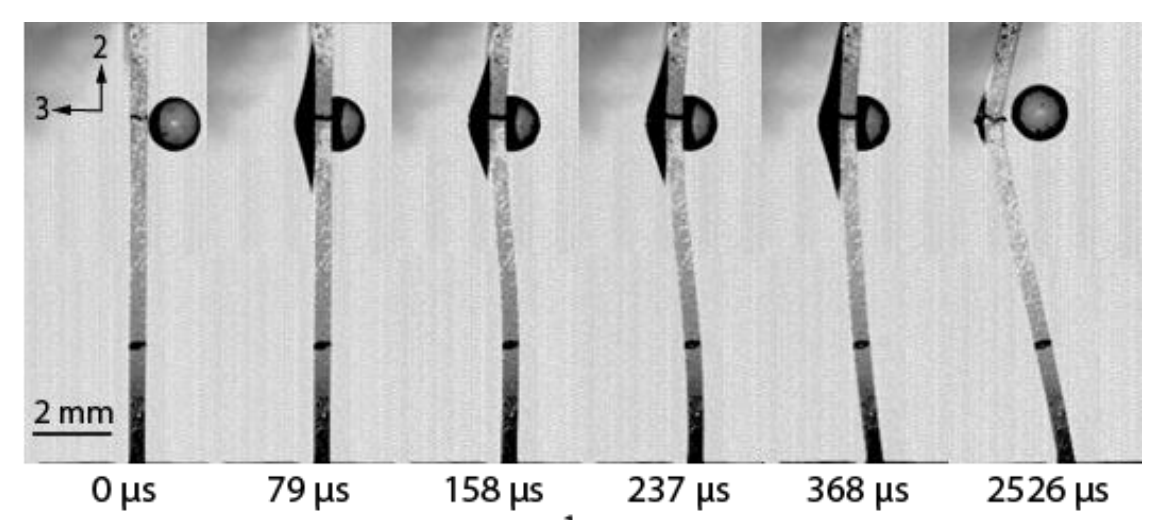

(a) $72 \mathrm{~ms}^{-1}$ Rebound

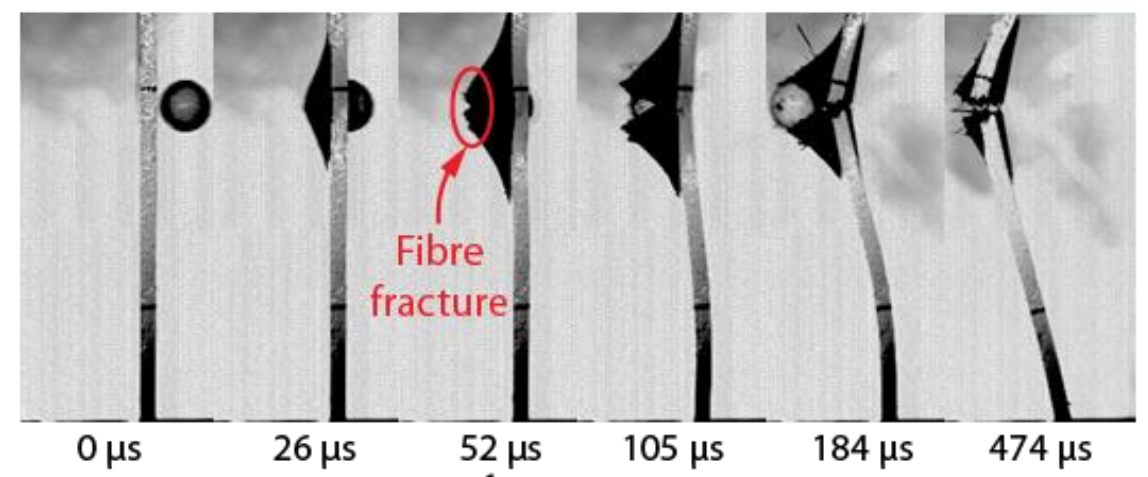

(b) $140 \mathrm{~ms}^{-1}$ _One-point fracture

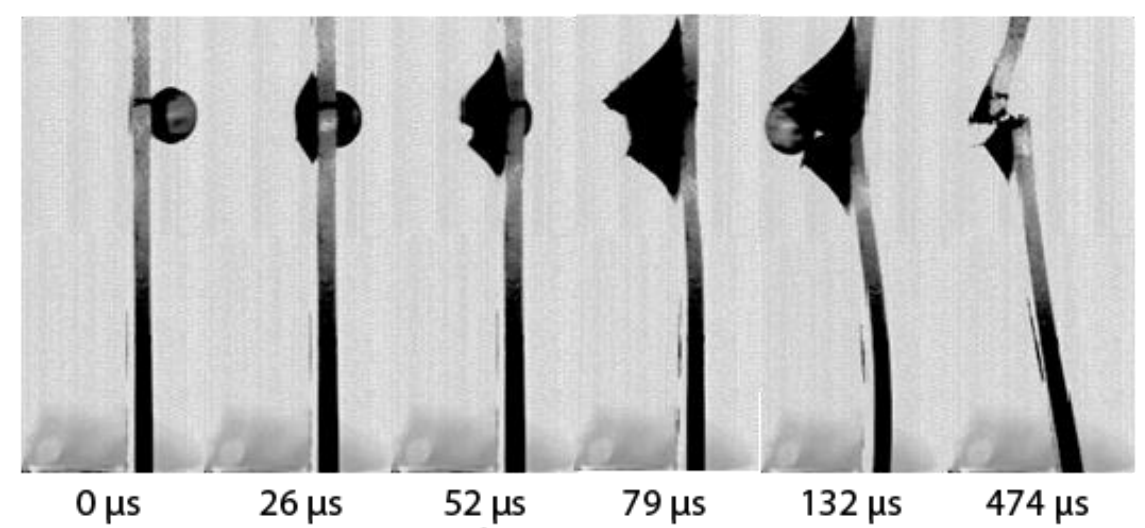

(c) $207 \mathrm{~ms}^{-1}$ _One-point fracture

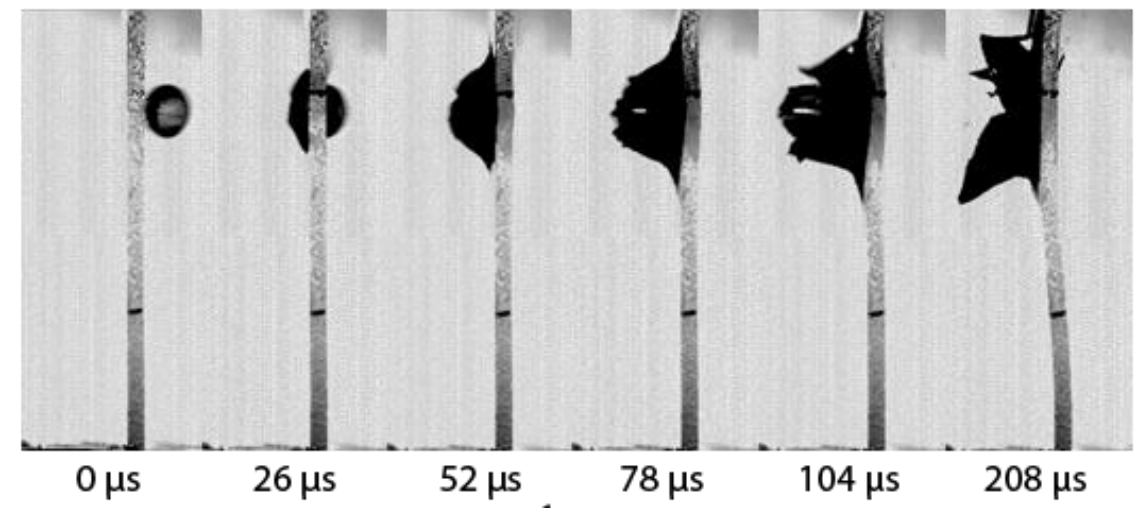

(d) $258 \mathrm{~ms}^{-1} \_$Perforation

Figure 7 


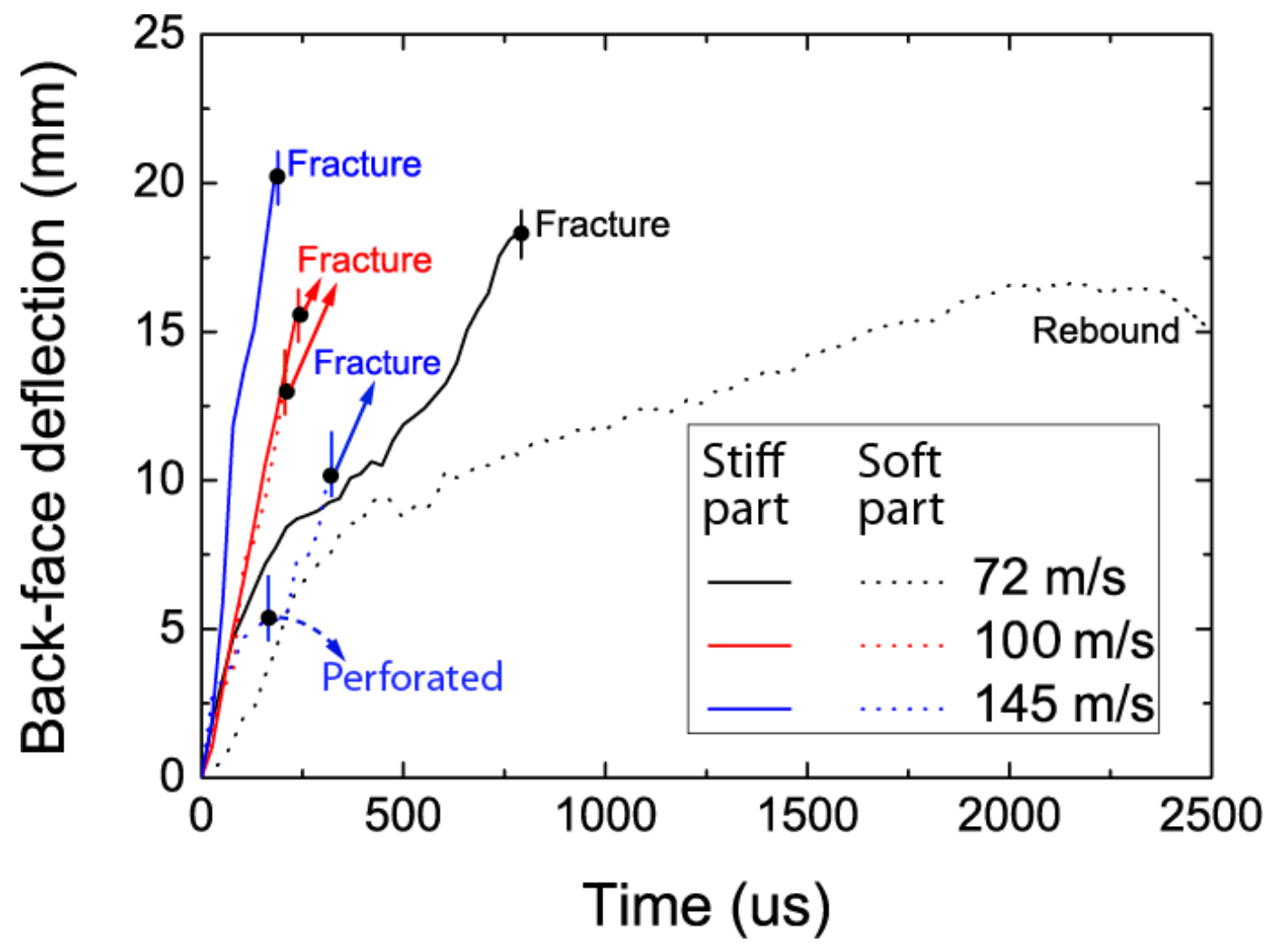

718

719

Figure 8

720

721

722

723 

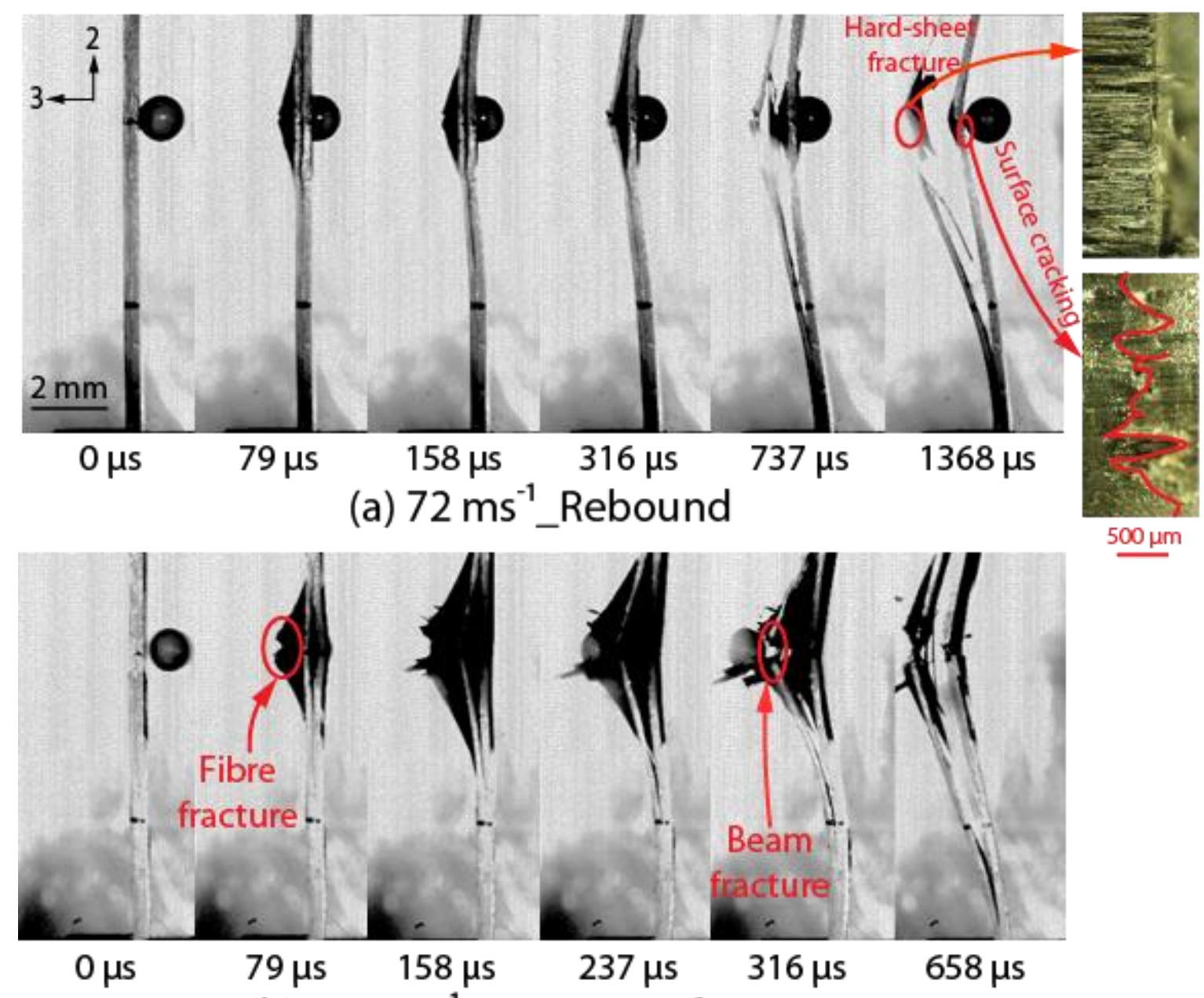

(b) $145 \mathrm{~ms}^{-1}$ _One-point fracture

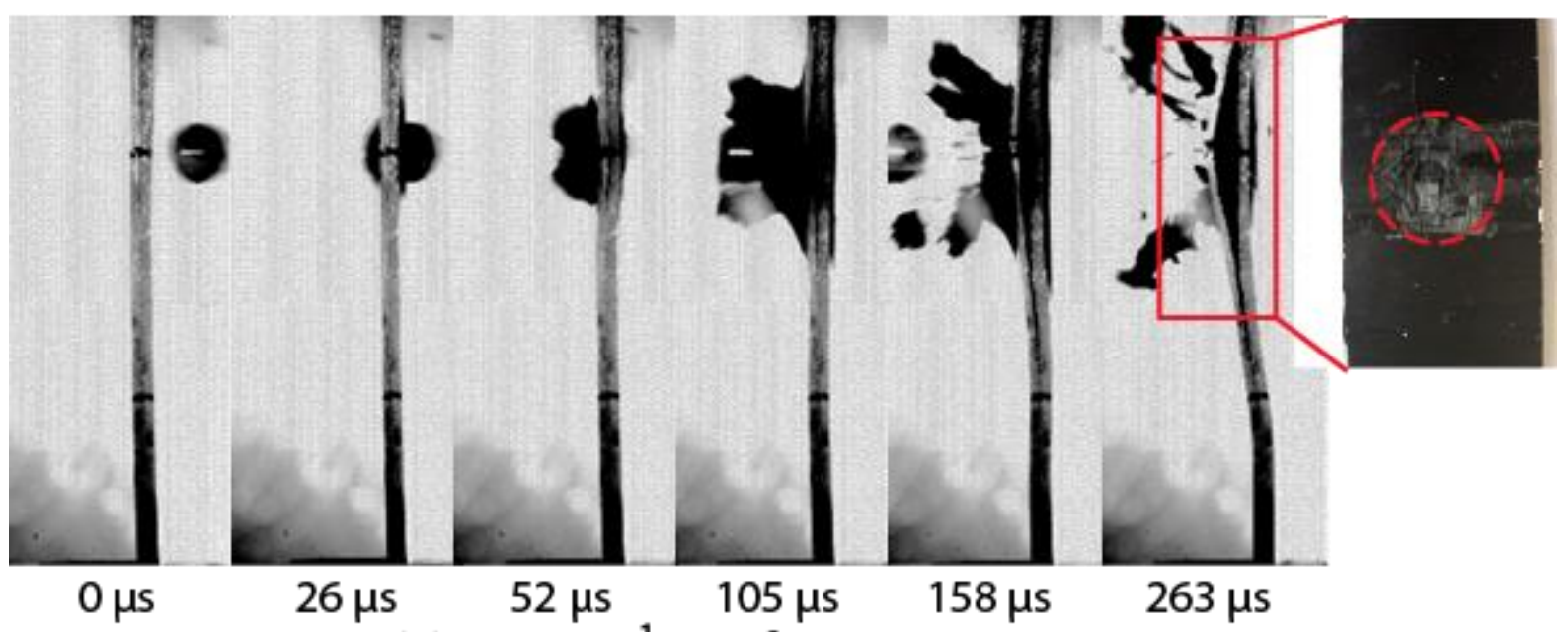

(c) $272 \mathrm{~ms}^{-1}$ _Perforation

Figure 9 


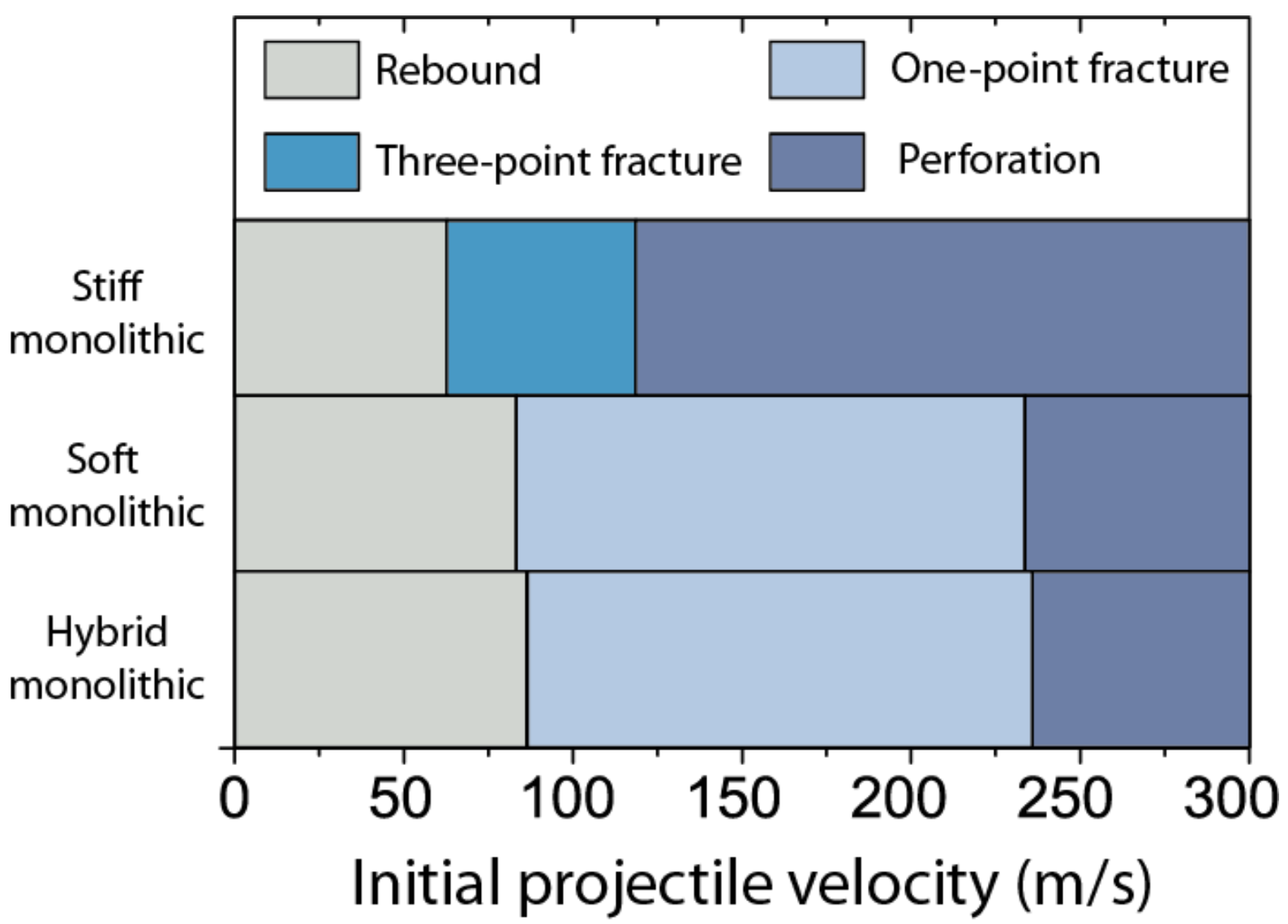

Figure 10 


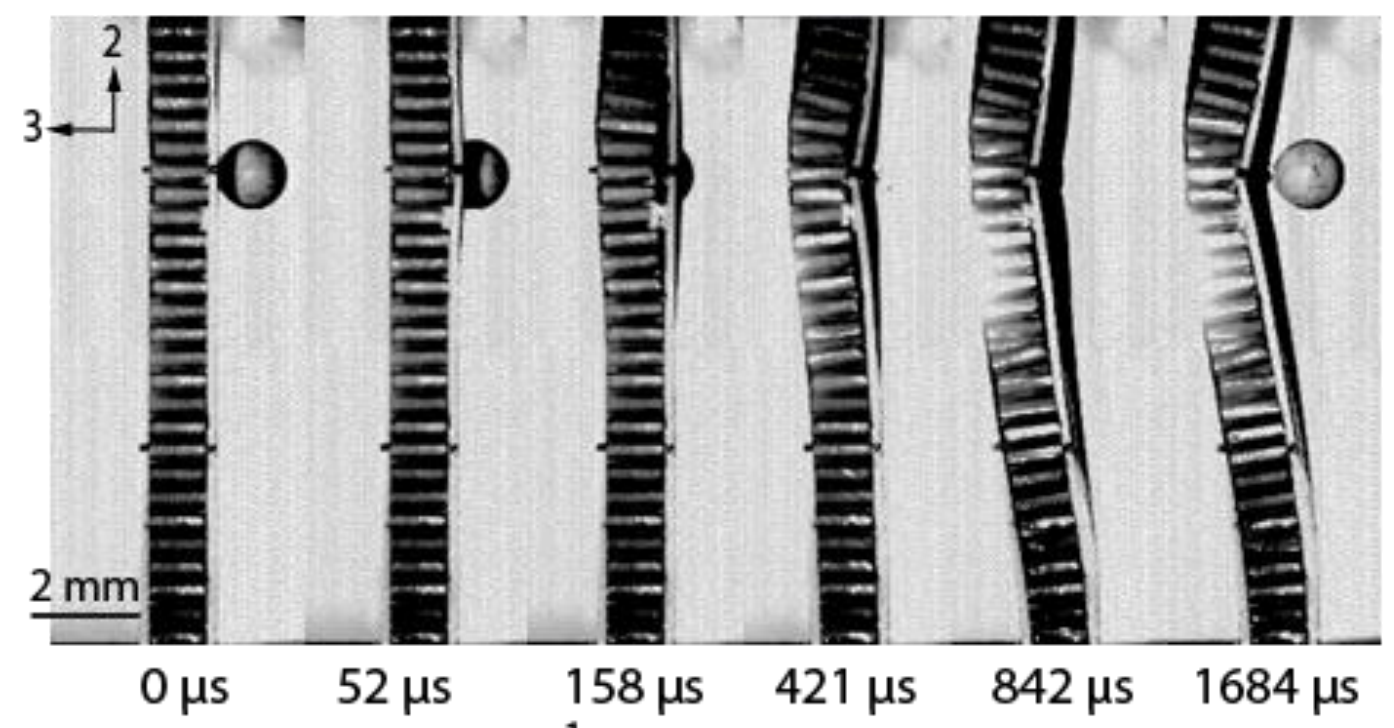

(a) $72 \mathrm{~ms}^{-1}$ _Stiff-face sandwich

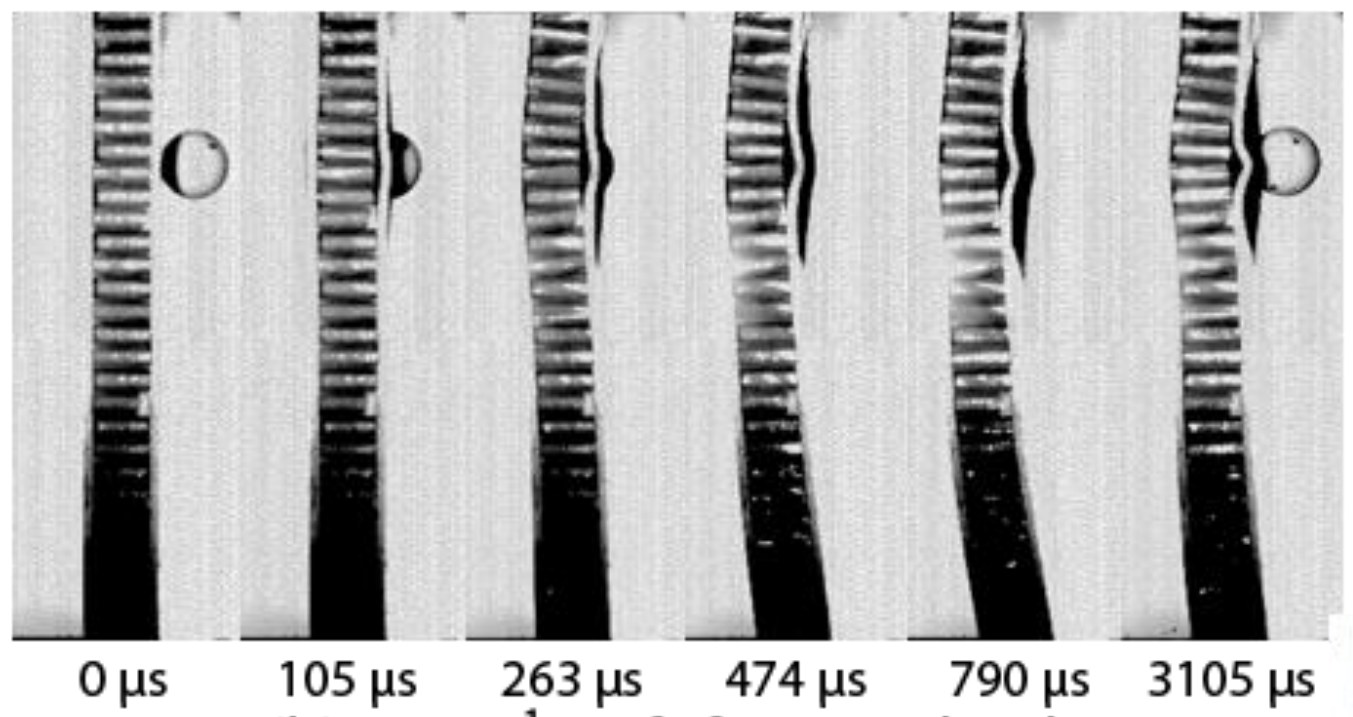

(b) $72 \mathrm{~ms}^{-1}$ _Soft-face sandwich

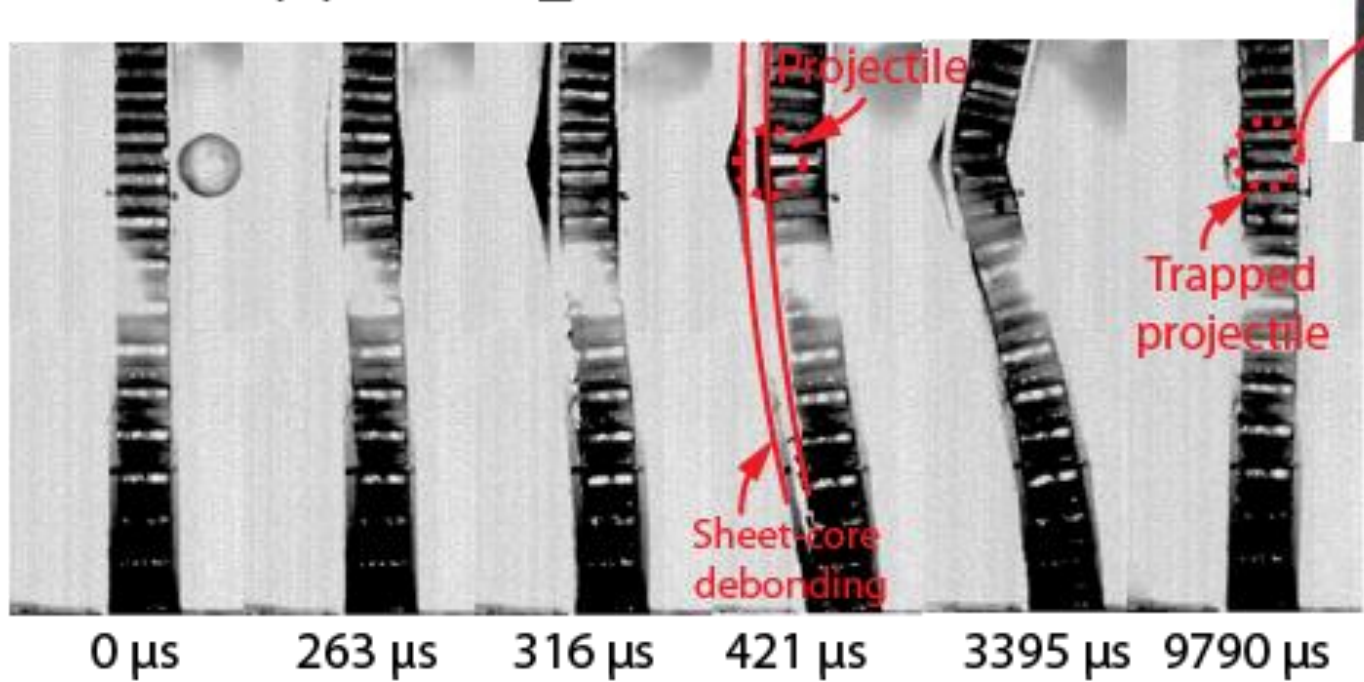

(c) $75 \mathrm{~ms}^{-1}$ _Hybrid-face sandwich

Figure 11 


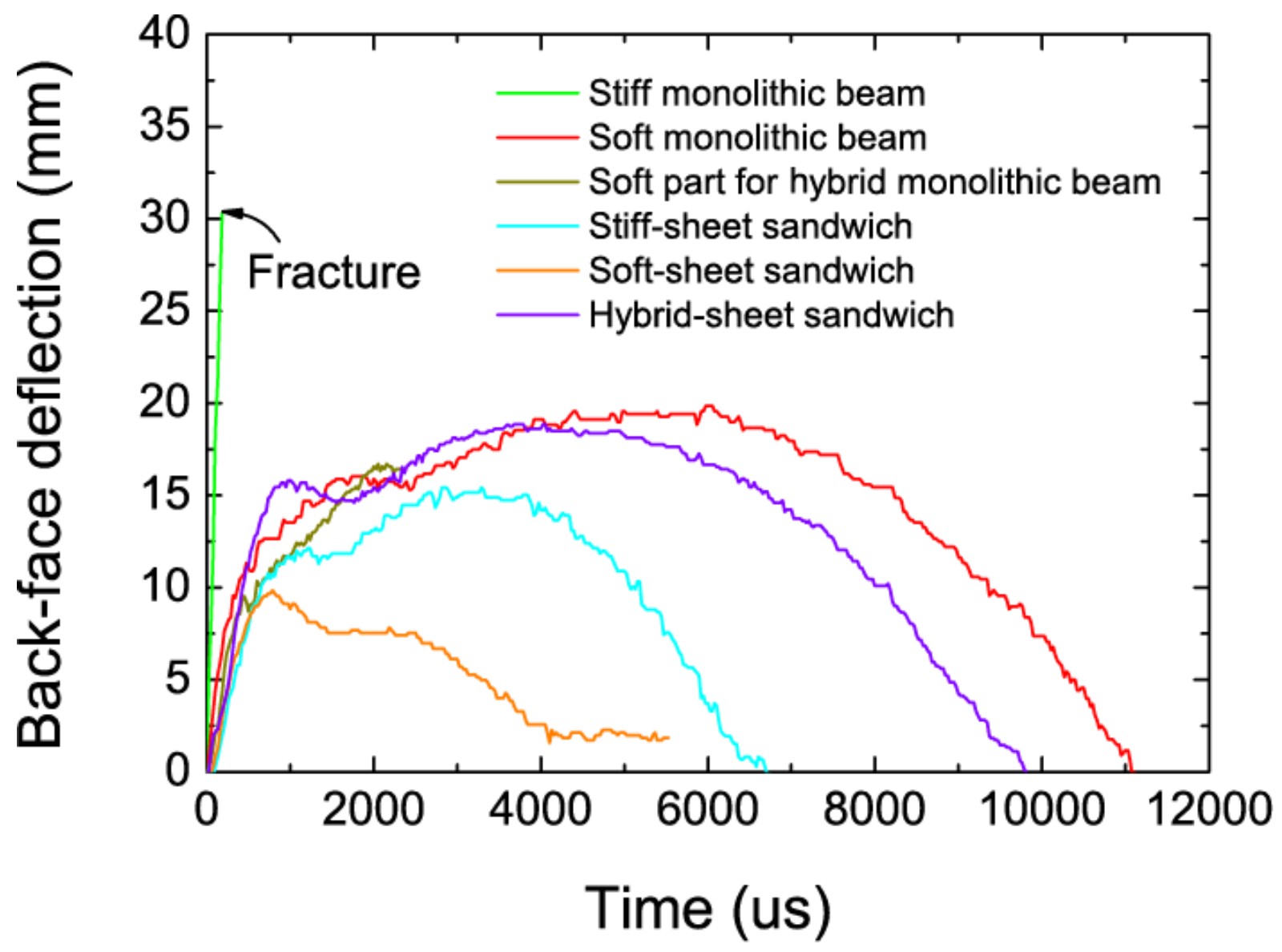

Figure 12 


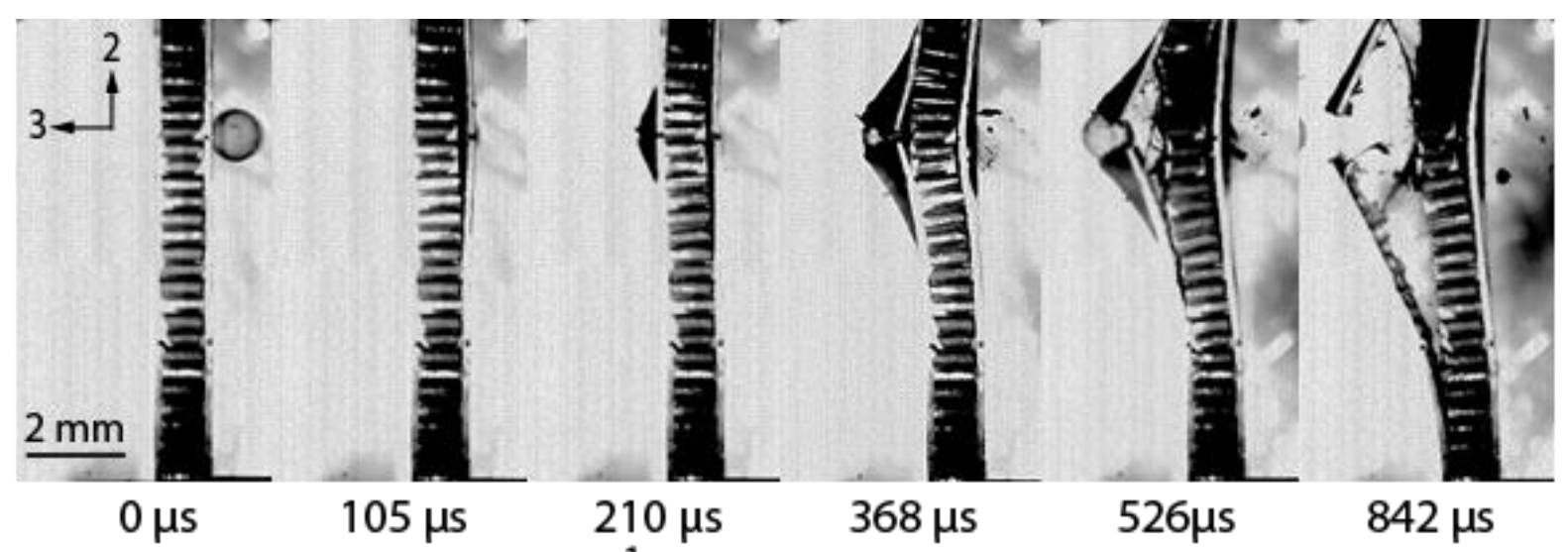

(a) $107 \mathrm{~ms}^{-1}$ _Stiff-face sandwich

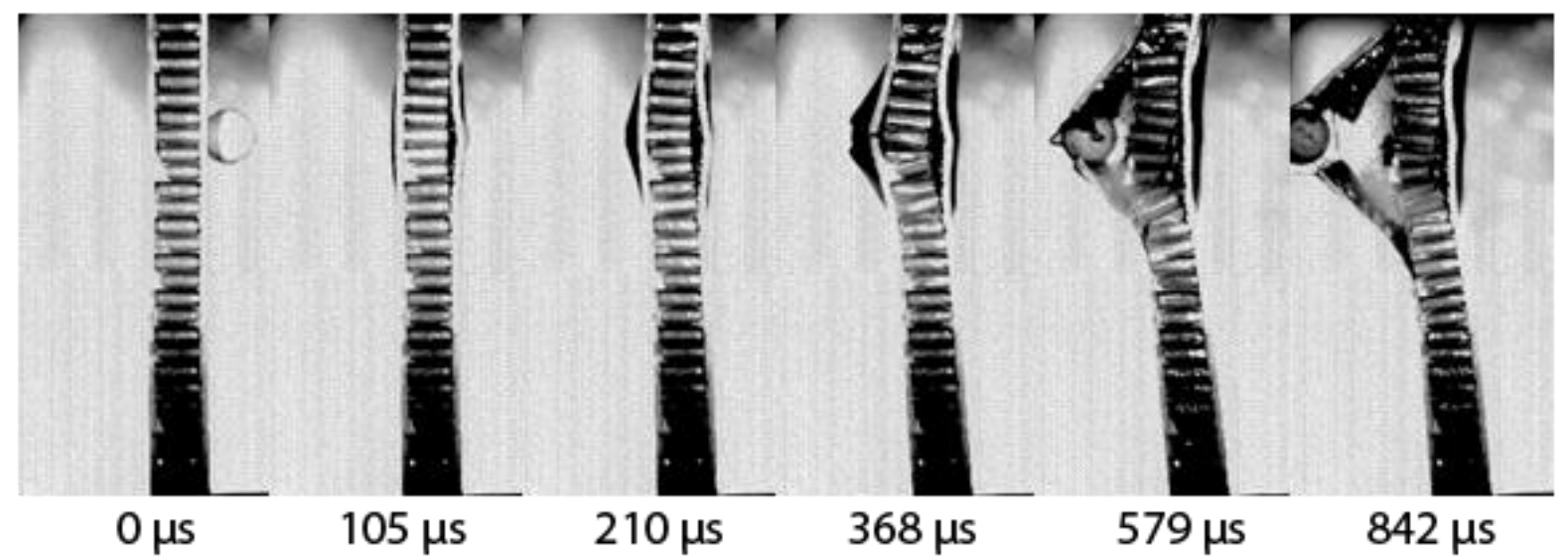

(b) $107 \mathrm{~ms}^{-1}$ _Soft-face sandwich

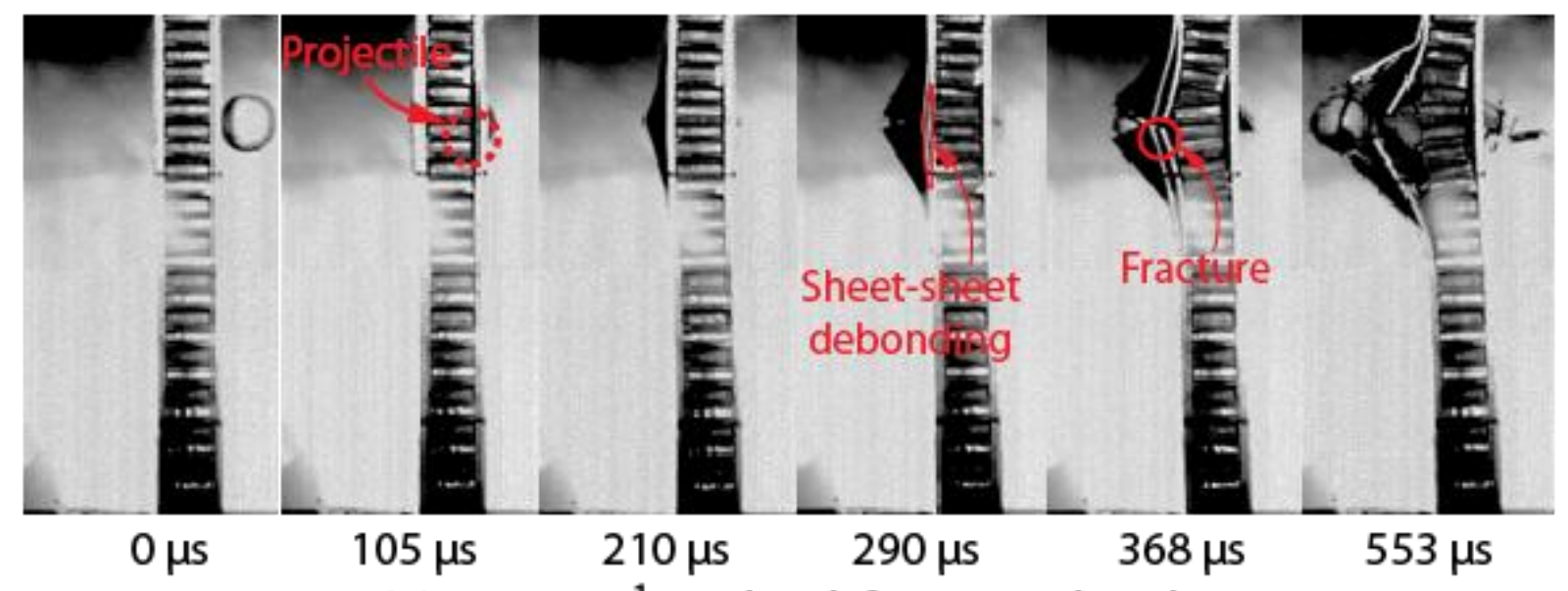

(c) $100 \mathrm{~ms}^{-1}$ _Hybrid-face sandwich

Figure 13 


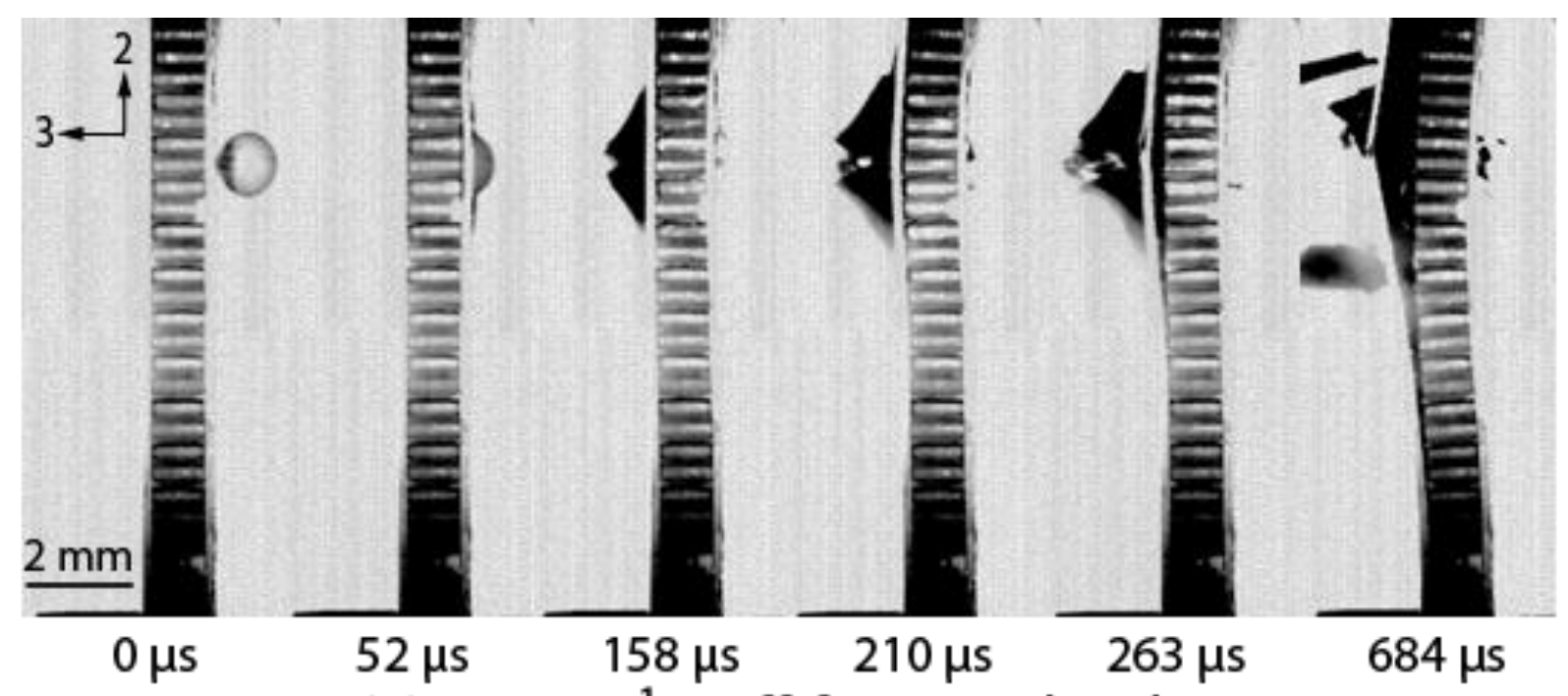

(a) $145 \mathrm{~ms}^{-1}$ _Stiff-face sandwich

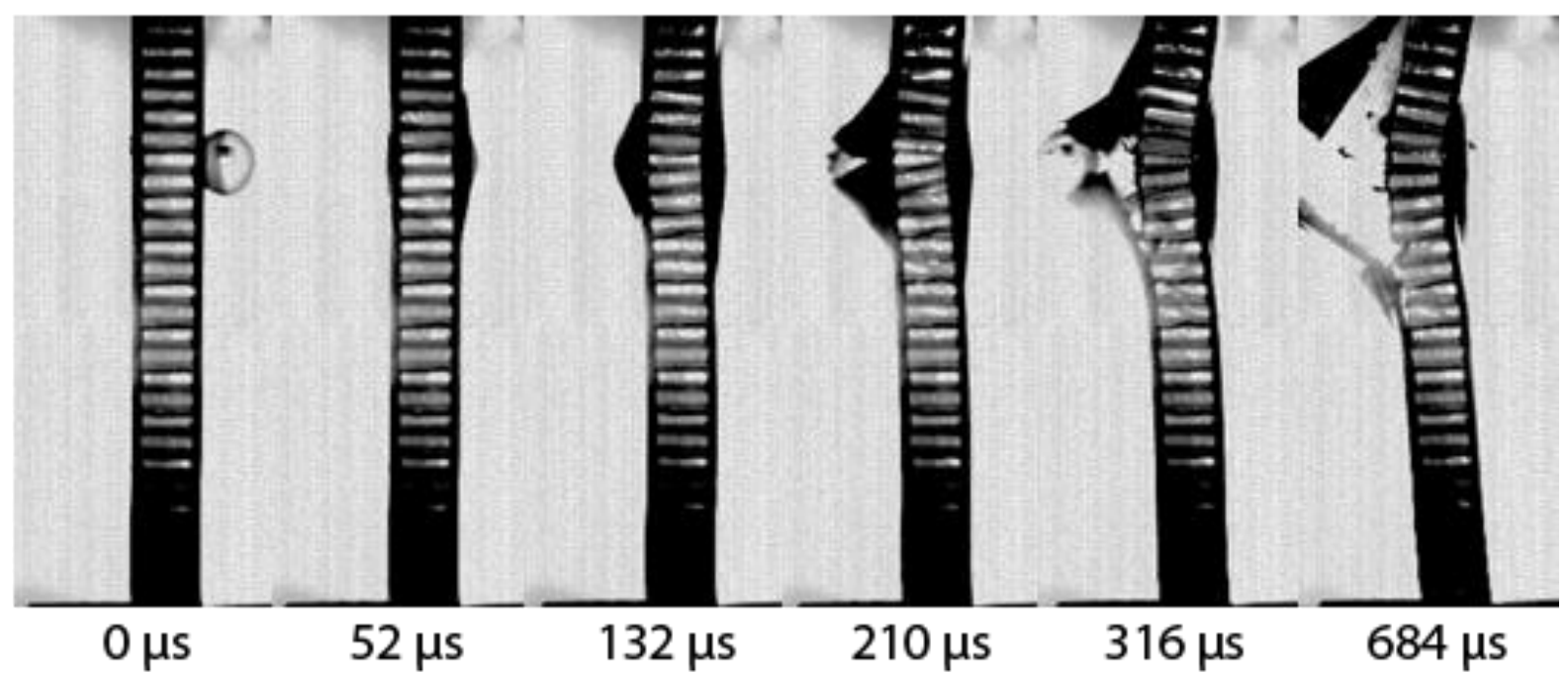

(b) $145 \mathrm{~ms}^{-1}$ _Soft-face sandwich

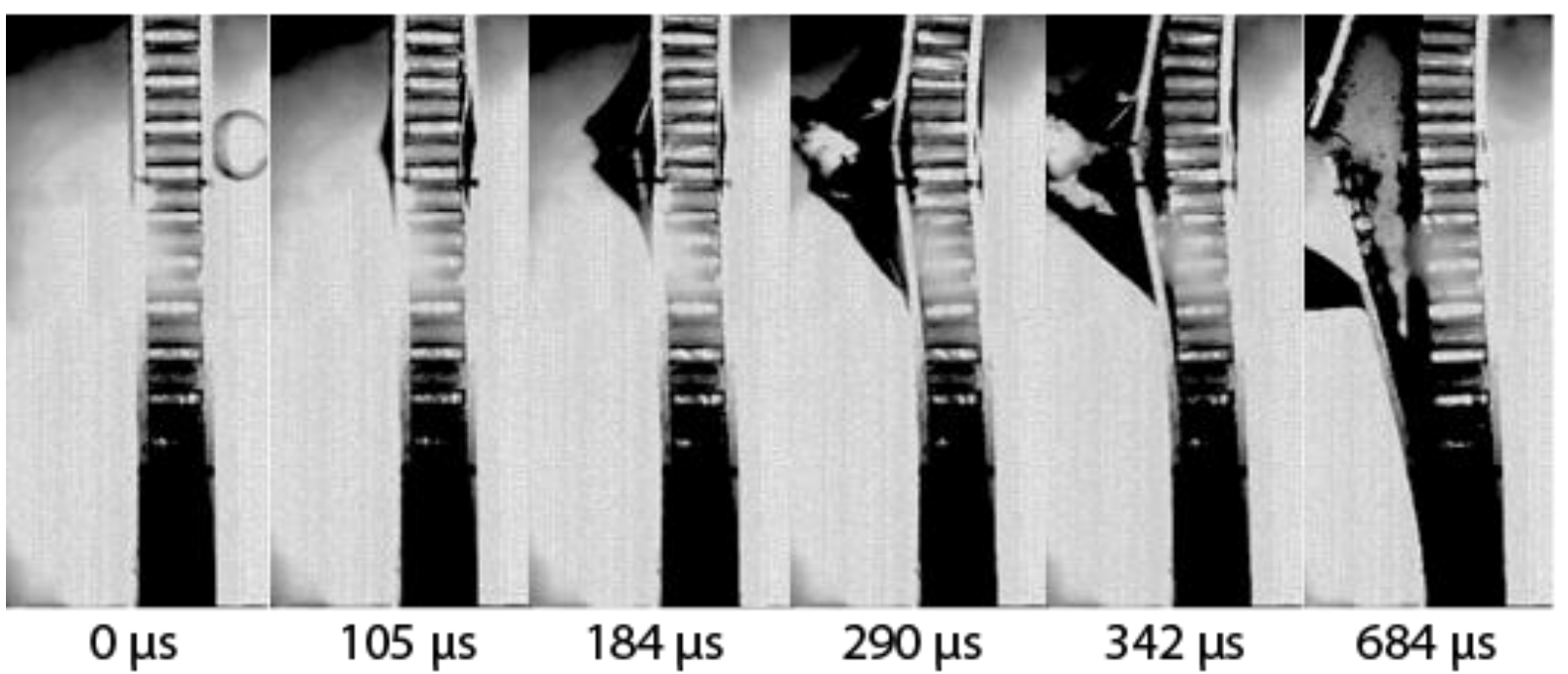

(c) $143 \mathrm{~ms}^{-1}$ _Hybrid-face sandwich

Figure 14 


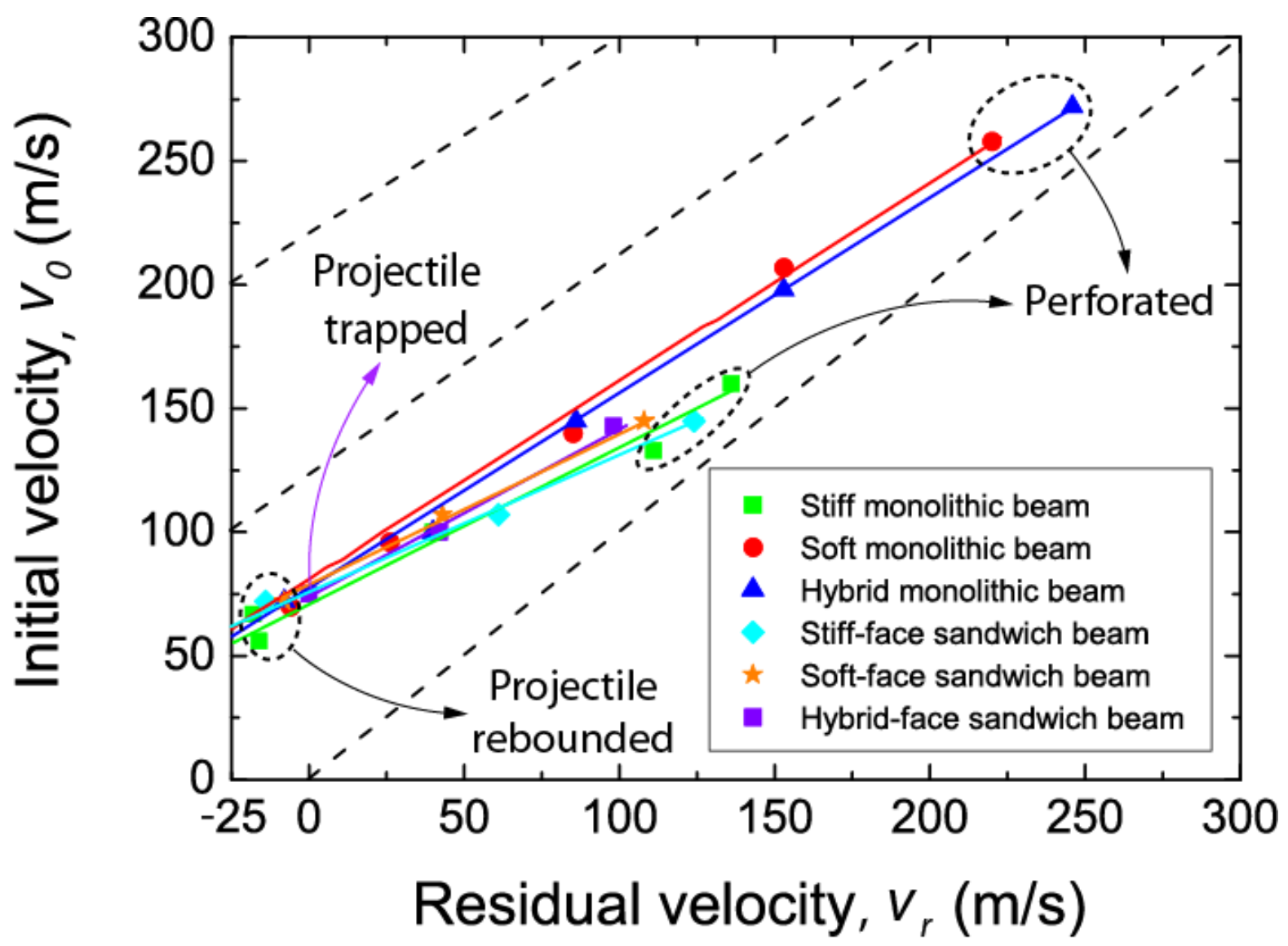

759

Figure 15 


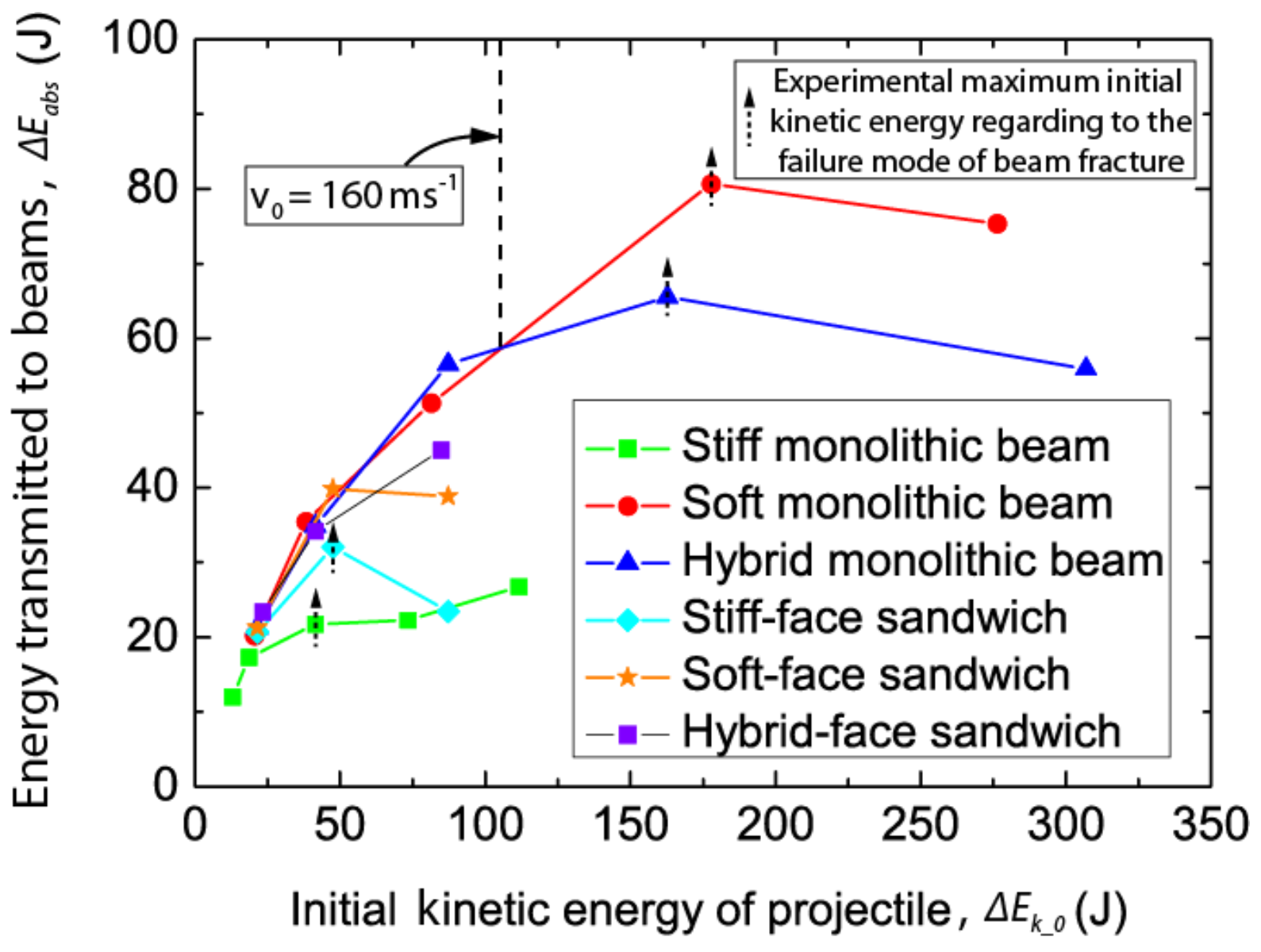

762

Figure 16 